\title{
Deep-ultraviolet nonlinear optical crystals by design: A computer-aided modeling blueprint from first principles
}

\author{
Lei Kang $^{1,2}$, Fei Liang ${ }^{1}$, Zheshuai Lin ${ }^{1^{*}}$ and Bing Huang ${ }^{2 *}$
}

\begin{abstract}
Deep-ultraviolet (DUV) nonlinear optical (NLO) crystal is indispensable in current DUV all-solid-state laser technology, which is the key device to generate DUV light by frequency conversion. Due to stringent criteria, DUV NLO crystals are scarce and their discovery faces a big challenge. Although $\mathrm{KBe}_{2} \mathrm{BO}_{3} \mathrm{~F}_{2}(\mathrm{KBBF})$ is already excellent for current uses, the development of DUV science requires the materials with shorter output wavelengths and larger NLO effects, so as to meet the needs of DUV lasers with higher precision and higher power. Therefore, how to efficiently design DUV NLO materials has always been the core issue in NLO materials science. Looking back on the development of NLO materials, it turns out that theoretical modeling and simulation is an effective and efficient method, not only for mechanism understandings, but also for experimental exploration. In this article, in order to accelerate the process of DUV materials discovery, we summarize and propose a powerful computeraided modeling system and design blueprint that can be used to evaluate the DUV NLO performance in a faster way than pure experiments. With this in hand, we enrich the understanding of NLO structure-property correlation, and systematically prospect the DUV NLO properties on the basis of many existing and designed structures according to different structural types and chemical compositions. Seven novel structures are predicted for the first time to exhibit potential DUV NLO capabilities. All the results enable us to believe that the computer-aided modeling blueprint will play an important role in the exploration of new DUV NLO crystals.
\end{abstract}

Keywords: deep-ultraviolet, nonlinear optical crystal, first-principles calculation

\section{INTRODUCTION}

Nonlinear optical (NLO) crystals have an irreplaceable role in the field of laser technology due to the advantages of high beam quality, tunable wavelengths, miniaturization and high power [1-4]. They also have a wide variety of applications in the field of information technology, such as quantum optics, optical phase modulation, and optical information storage [5-9]. As such, NLO crystals are important fundamental materials for optoelectronic information technology today and in the future. Remarkably, in the deep-ultraviolet (DUV, $\lambda<200 \mathrm{~nm}$ ) region, NLO crystals play a key role in developing the DUV lasers for photolithography, atto-second pulse generation, and advanced instrument development, etc. [10-14]. An important example is ultra-precision angular resolved photoelectron spectroscopy (ARPES) that adopts the $177.3 \mathrm{~nm}$-laser, which has been widely used in the surface state measurements of high-temperature superconductors, topological insulators and two-dimensional (2D) materials [14-17].

The core and key issue in the field of NLO crystals is to improve the comprehensive performance of NLO crystals. For fulfilling the higher DUV conversion, they must comprise two stringent criteria, i.e., short phase-matching (PM) wavelength and high second-harmonic generation (SHG) efficiency [18-20]. In general, a qualified DUV NLO crystal must have (I) good DUV transparency, requiring optical bandgap $E_{\mathrm{g}} \geq 6.3 \mathrm{eV}$ and preferably $\geq$ $7.1 \mathrm{eV}$ for the practical $177.3 \mathrm{~nm}$-laser; (II) sufficient PM capacity, usually requiring large DUV birefringence with $\Delta n>0.08$ in the DUV region. The shortest PM wavelength $\lambda_{\mathrm{PM}}$ depends on the dispersion of respective re-

\footnotetext{
${ }^{1}$ Technical Institute of Physics and Chemistry, Chinese Academy of Sciences, Beijing 100190, China

${ }^{2}$ Beijing Computational Science Research Center, Beijing 100193, China

* Corresponding authors (emails: zslin@csrc.ac.cn (Lin Z); bing.huang@csrc.ac.cn (Huang B))
} 
fractive indices, e.g., $n_{\max }\left(2 \lambda_{\mathrm{PM}}\right)=n_{\min }\left(\lambda_{\mathrm{PM}}\right)$ for type-I PM process; and (III) effective SHG effect $d_{\text {eff }}$, determined by large SHG coefficient $\left|d_{i j}\right| \geq d_{36}=0.39 \mathrm{pm} \mathrm{V}^{-1}$ of $\mathrm{KH}_{2} \mathrm{PO}_{4}$ (KDP) in the PM direction, and in general, the larger $d_{i j}$, the higher conversion efficiency. Only meeting the criteria of (I), (II) and (III), can crystals realize PM DUV conversion. To date, few crystals can satisfy these criteria because of either small $\Delta n$ (e.g., $\left.\mathrm{LiB}_{3} \mathrm{O}_{5}(\mathrm{LBO})\right)$ or $E_{\mathrm{g}}$ (e.g., $\beta-\mathrm{BaB}_{2} \mathrm{O}_{4}(\mathrm{BBO})$ ) [21-25]. In this sense, the realization of DUV conversion needs good balance of $E_{\mathrm{g}} v s . \Delta n$ with not only sufficiently wide DUV $E_{\mathrm{g}}$ but also sufficiently large DUV $\Delta n$. Under the premise of this balance, large SHG effects are then favorable.

In fact, for most structures it is difficult to achieve the rigorous balance, making DUV NLO crystals extremely scarce. As a rare exception, $\mathrm{KBe}_{2} \mathrm{BO}_{3} \mathrm{~F}_{2}(\mathrm{KBBF})$ has a structural feature beneficial for this balance [26], exhibiting a large birefringence $(\Delta n \approx 0.08)$, strong SHG effect $\left(d_{22} \approx 0.5 \mathrm{pm} \mathrm{V}^{-1}\right)$, wide bandgap $\left(E_{\mathrm{g}} \approx 8.3 \mathrm{eV}\right)$ and good mechanical stability. For current uses, KBBF is already an excellent DUV NLO crystal, which has practically achieved the 177.3-nm laser with $200 \mathrm{~mW}$ and has played a very important impetus in the DUV laser fields $[3,26,27]$. It should be emphasized that, owing to the difficulty of crystal growth, the thickness of KBBF along the $z$-axis is limited to $2-3 \mathrm{~mm}$, making the power improvement at $177.3 \mathrm{~nm}$ very small. The development of DUV field requires that NLO crystals have shorter PM wavelengths and stronger SHG effects, so as to meet the increasingly urgent needs for DUV lasers with higher precision and higher power [13]. Therefore, it is necessary to explore more promising DUV NLO crystals to achieve this goal.

However, no material was found to exceed the performance of KBBF before 2013 [22]. This is mainly because KBBF has pushed the DUV NLO performance to the extreme in borates based on the anionic group theory proposed by Prof. Chuangtian Chen [21]. This theory captures the essence of NLO origin and provides a concise structure-property law for UV/DUV NLO crystals, which has indeed achieved a great success in guiding the discovery of BBO, LBO and KBBF, but it is inefficient to evaluate the NLO performance in an ab-initio manner due to the approximation that ignores the contribution of $A$-site cations. Therefore, as the anionic group theory faces the challenge from the stringent criteria for accurate evaluation [28], the improvement of DUV NLO performance is an arduous task in NLO materials science.

With the in-depth understanding of NLO mechanisms, high-precision first-principles methods have been adop- ted into the studies of NLO crystals in the past two decades [18]. A complete set of CASTEP-compatible programs based on density functional theory (DFT) calculations have been successfully developed, which have advanced the NLO crystal field and accelerated the experimental explorations [29-34]. The proposed firstprinciples method for NLO calculations is based on strict crystal band and perturbation theory, without empirical parameter settings. As such, it is more efficient to accurately obtain the NLO properties, and more reliable to $a b$ initio design new NLO structures [28].

It is worth noting that, combining theoretical predictions with experimental characterizations, some compounds have been obtained, which do exhibit DUV NLO properties comparable to that of KBBF. As the first breakthrough in 2013, our group proposed a series of DUV NLO fluoride carbonates [35], in which $\mathrm{RbAlCO}_{3} \mathrm{~F}_{2}$ was predicted with wide $E_{\mathrm{g}}$ and large $\Delta n$ so as to induce $\lambda_{\mathrm{PM}} \approx 152 \mathrm{~nm}$. This is the first time to predict possible DUV NLO structures beyond KBBF theoretically. In 2015, two novel DUV NLO structures, i.e., $\mathrm{NH}_{4} \mathrm{Be}_{2} \mathrm{BO}_{3} \mathrm{~F}_{2}$ (ABBF) and $\gamma-\mathrm{Be}_{2} \mathrm{BO}_{3} \mathrm{~F}(\gamma-\mathrm{BBF})$, were theoretically predicted [36]. Both of them were synthesized experimentally in 2018 [37], with good agreement between experimental and predicted results. Other achievements were completed in 2017-2018 [38-43]. And especially, $\mathrm{NH}_{4} \mathrm{~B}_{4} \mathrm{O}_{6} \mathrm{~F}(\mathrm{ABF})$ series with excellent DUV NLO properties were synthesized [40]. These could originate from the first-principles studies for $\mathrm{LiB}_{6} \mathrm{O}_{9} \mathrm{~F}$ series from 2011 to 2013 [44]. And the experiments in 2017 awakened the research of the fluorooxoborates [38].

These new developments clearly demonstrate that the search for DUV materials has not approached to the extreme, but only encounters the "bottleneck". Inspired by the Materials Genome Project [45], we believe that there still have room for the discovery of new DUV NLO structures by combining different NLO-active "genes" beyond $\left(\mathrm{BO}_{3}\right)^{3-}$ and $\left(\mathrm{BeO}_{3} \mathrm{~F}\right)^{5-}$ in $\mathrm{KBBF}$. The computeraided simulations are playing an increasingly important role in the design and search for more promising DUV NLO materials other than KBBF, and the theoretical research on structure-property relations will become more efficient to promote to satisfy criteria (I), (II) and (III).

In this work, we design a computer-aided modeling system for DUV NLO crystals by adopting the KBBF structure as the starting point. By adopting first-principle methods and high-throughput proposal, this modeling system considers different structure types and chemical compositions, and comprehensively includes the contribution of anionic and cationic parts to DUV NLO 
performance to accurately evaluate the DUV NLO properties of many existing and designed compounds. Using this system, we indeed find several new structures exhibiting potential DUV NLO capabilities, including wide energy bandgap, strong SHG effect, large birefringence and short PM output wavelength. Therefore, this computer-aided modeling system provides an efficient and effective guidance for exploring DUV NLO materials, by avoiding the waste of human and financial resources.

\section{COMPUTATIONAL SECTION}

Different from the experiments, the computer-aided modeling system can provide efficient benefits to evaluate the energy bandgap, SHG effect, birefringence and PM output by a reasonable, controllable and convenient program. Fig. 1 depicts the flowchart of probing DUV NLO crystals based on KBBF, which is basically the starting structure for most DUV modeling. Herein, KBBF can be divided into four components according to microscopic structure units and chemical compositions, i.e., $A$-site cation $\mathrm{K}^{+}, B$-site cation $\mathrm{Be}^{2+}, G$ (group)-site anionic group $\left(\mathrm{BO}_{3}\right)^{3-}$, and $H$ (halogen)-site halogen anion $\mathrm{F}^{-}$.

The first step needs a new compound with the general chemical formula of $A_{i} B_{j} G_{m} H_{n}(i, j, m, n=0,1,2 \ldots)$ through rational substituents of $A$-site, $B$-site, $G$-site, and/ or $H$-site ions or groups. The $A$-site cations mainly include those cations with strong ionic characteristics, such as alkali and alkaline earth metal cations; the $B$-site cations mainly include the center ions usually coordinated with oxygen atoms, such as $\mathrm{Be}^{2+}, \mathrm{Zn}^{2+}, \mathrm{Al}^{3+}, \mathrm{Sc}^{3+}$; the Gsite anionic groups mainly include covalent oxide units such as $\left(\mathrm{BO}_{3}\right)^{3-},\left(\mathrm{BO}_{4}\right)^{5-},\left(\mathrm{CO}_{3}\right)^{3-},\left(\mathrm{NO}_{3}\right)^{-},\left(\mathrm{SiO}_{4}\right)^{4-}$, $\left(\mathrm{PO}_{4}\right)^{3-},\left(\mathrm{SO}_{4}\right)^{2-}$ and their combinations; and the $\mathrm{H}$-site halogen anions mainly refer to $\mathrm{F}^{-}, \mathrm{Cl}^{-}$, etc.

The second step needs to design initial structures based on the selection principles. To satisfy the criterion (I), oxides/halides are the first selection option since other compound systems are difficult to possess a DUV $E_{\mathrm{g}}$. The ions should not include non-filling $\mathrm{d}$ or $\mathrm{f}$ electrons (e.g., $\left.\mathrm{Ce}^{3+}\right)$, electronic lone pairs (e.g., $\mathrm{Bi}^{3+}$ ), and large covalent radius (e.g., $\mathrm{Cd}^{2+}$ ), and the nonbonding states should be removed as much as possible. For criterion (II), layerstructures are better than 3D frame-structures (e.g., $\mathrm{BPO}_{4}$ ) due to larger anisotropy. Moreover, layer-structures usually have more moderate refractive dispersions, especially in the spectral region close to $E_{g}$, which are more suitable to achieve the $\mathrm{PM}$ condition compared with chain-structures (e.g., $\mathrm{NaBeB}_{3} \mathrm{O}_{6}$ and $\mathrm{LiB}_{6} \mathrm{O}_{9} \mathrm{~F}$ ) [46]. Criterion (III) is not harsh since many acentric compounds

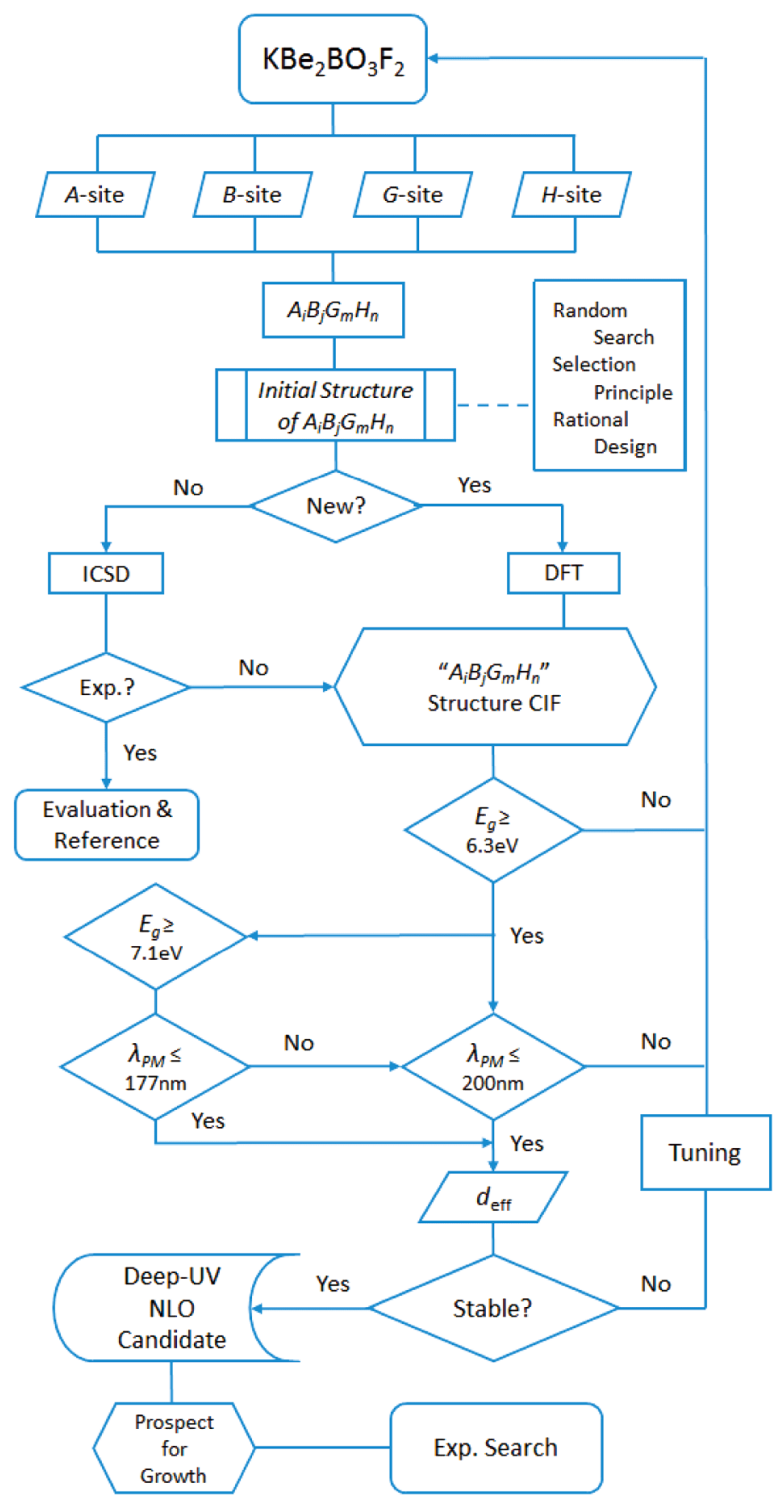

Figure 1 Computer-aided modeling system and design blueprint of DUV NLO crystals based on the KBBF system.

with parallel polar units can have a relatively strong SHG effect $(\sim \mathrm{KDP})$. It is accordingly summarized that the structure-selection principles for DUV NLO materials comprise: (i) a layer-structure with polar-parallel alignment, (ii) active-groups without nonbonding orbitals, and (iii) suitable connections between active-groups. Taking $\left(\mathrm{BO}_{3}\right)^{3-}$ as an example, (a) $\left(\mathrm{BO}_{3}\right)^{3-}$ should remain coplanar; (b) three terminal oxygens should be attached to covalent atoms, e.g., $\mathrm{Be}^{2+}$ or $\mathrm{Al}^{3+}$; (c) anionic-group-density should be as high as possible [47]. This is actually the reason why KBBF exhibits such predominant DUV NLO performances by well satisfying these principles. 
Once the initial structures of $A_{i} B_{j} G_{m} H_{n}$ are obtained, our targeted calculations can be employed to run the third step to determine whether the candidates are good or not. If the structure has already existed in the crystal database, e.g., ICSD (Inorganic Crystal Structures Database), its NLO results can be naturally obtained from references or calculations. If the structure is new, the following procedure is to perform the high-precision first-principles calculations for the structural optimization and electronic structures. In our first-principles calculations, the plane wave pseudopotential method implemented in CASTEP based on DFT was adopted $[48,49]$. The ion-electron interactions are modeled by the optimized norm-conserving pseudopotentials [50]. The crystal geometries are optimized using the quasi-Newton method [51], followed by the band structure and optical properties calculations. According to our examinations for DUV oxides, the hybrid PBE0 functional was used to determine $E_{\mathrm{g}}$, while the scissors-corrected GGA method with PBE functional was used to calculate the optical properties, wherein the scissors-operator is the difference between PBE0 and GGAPBE bandgaps $[32,52,53]$. After the determination of refractive indices $n$ and birefringence $\Delta n$, the shortest PM wavelength $\lambda_{\mathrm{PM}}$ can be computed. Meanwhile, using the expressions developed by Lin et al. [30], the SHG coefficients $d_{i j}$ can be simulated. As a self-consistent $a b$ initio approach without introducing any experimental parameter, our computational method has been proven to be very suitable to study the NLO properties in DUV NLO materials [34].

In order to obtain the most favorable structures, necessary judgments must be performed. The first step is to determine whether $E_{\mathrm{g}} \geq 6.3 \mathrm{eV}$, i.e., $\lambda_{\mathrm{UV}} \leq 200 \mathrm{~nm}$. If the result is "Yes", it would be better to judge whether $E_{\mathrm{g}} \geq$ $7.1 \mathrm{eV}$, i.e., $\lambda_{\mathrm{UV}} \leq 177.3 \mathrm{~nm}$ for the sixth harmonic generation (6HG) of $1064 \mathrm{~nm} \mathrm{Nd:YAG} \mathrm{lasers.} \mathrm{The} \mathrm{second}$ step is to determine whether $\lambda_{\mathrm{PM}} \leq 200$ or $177 \mathrm{~nm}$, which is the most critical evaluation for the desired DUV output. The third step is to give the effective SHG coefficient $d_{\text {eff }}$ under type I or II PM condition by using the obtained matrix of SHG coefficients $d_{i j}$, and to estimate the conversion efficiency under ideal conditions. If the structure exhibits good DUV NLO performance, the next step would be the evaluation of the prospect for single crystal growth and post-processing. The dynamic stability of the compound structure can be determined by the calculated phonon dispersion spectrum which has virtual frequency or not [54]. Meanwhile, the lattice stability under ambient temperature can be confirmed by molecular dynamics (MD) simulations [55]. If the structure is maintained after a long-time MD simulation, it would exhibit practically stable properties. In addition, the elastic and thermal properties can be theoretically calculated if necessary. With the comprehensive DFT evaluation, it is anticipated that the experimental synthesis and characterizations would be significantly accelerated.

The present DFT calculations are sufficiently effective and can be more efficient than conventional experimental explorations, in which bulk crystals with suitable size and good quality are necessary for the measurements of DUV $E_{\mathrm{g}}$ (e.g., UV-vis diffuse reflection method), SHG coefficients (e.g., Maker fringes method) and refractive indices (e.g., polarizing microscope method). The large-size crystal growth is complicated and time-consuming, which hinders the rapid characterizations of practical NLO properties. Although some preliminary experiments can be employed for evaluation, the evidences are not adequate and the errors might be large. Following our extensive studies and testing, in a highly accuracy way, we can efficiently obtain the bandgap $E_{\mathrm{g}}$, SHG coefficients $d_{i j}$, birefringence $\Delta n$, the shortest SHG output $\lambda_{\mathrm{PM}}$ and finally effective SHG effect $d_{\text {eff, }}$ all of which are the most necessary index for determining DUV NLO performance. In addition, the mechanical and crystal growth property can be compared with KBBF based on the calculations, such as interlayer binding energy, thermodynamic properties, and mechanical-elastic constants. From the analysis, the structure-property relationships can be obtained to guide the consequent researches.

\section{RESULTS AND DISCUSSION}

The core step of searching for DUV NLO crystals is to produce a rational structure that fits the DUV criteria. Based on the modeling system and structure-selection principles, we attempt to design such kinds of NLO structures. We discuss almost-all kinds of possible compounds of $A_{i} B_{j} G_{m} H_{n}$, in which potential DUV NLO crystals might emerge from combined theoretical analysis and available experimental comparisons. These existing and designed compounds are mainly examined in five ways, i.e., reasonable $A$-site substitute, $B$-site substitute, $G$-site substitute, $H$-site substitutes, and without $A$-site cations, respectively. The five design strategies include both the cationic and anionic contributions to the NLO performance, which will provide a more comprehensive understanding on the structure-property correlation of DUV NLO crystals.

\section{$A$-site cationic substitute}

The $A$-site cations herein include alkali-metals (e.g., $\mathrm{Li}^{+}$, 
$\mathrm{Na}^{+}, \mathrm{K}^{+}, \mathrm{Rb}^{+}, \mathrm{Cs}^{+}$), alkaline-earth-metals (e.g., $\mathrm{Ca}^{2+}, \mathrm{Sr}^{2+}$, $\mathrm{Ba}^{2+}$ ), and some cationic groups (e.g., $\mathrm{NH}_{4}^{+}, \mathrm{CH}_{3} \mathrm{NH}_{3}^{+}$). Based on the anionic group theory, the $d_{i j}$ or $\Delta n$ is mainly derived from the anionic groups with large geometrical anisotropy. The spherical $A$-site cations have negligible contribution to the overall SHG effect. However, they can change the micro-arrangement and adjust the final cellsize, to some extent affecting the ultimate NLO properties.

$\mathrm{ABe}_{2} \mathrm{BO}_{3} \mathrm{~F}_{2}\left(\mathrm{~A}=\mathrm{K}, \mathrm{Rb}, \mathrm{Cs} ; \mathrm{Na}, \mathrm{Li}, \mathrm{Tl} ; \mathrm{NH}_{4}, \mathrm{CH}_{3} \mathrm{NH}_{3}\right)$

As typical KBBF-family members, $\mathrm{RbBe}_{2} \mathrm{BO}_{3} \mathrm{~F}_{2}$ (RBBF) and $\mathrm{CsBe}_{2} \mathrm{BO}_{3} \mathrm{~F}_{2}(\mathrm{CBBF})$ have similar topological structures as KBBF (Fig. 2a) [56-58]. Table 1 lists their crystallographic data with good agreement between experimental (exp.) and calculated (cal.) results. As the cationic size increases, $\left(\mathrm{BO}_{3}\right)^{3-}$ remains the same size but $\left(\mathrm{BeO}_{3} \mathrm{~F}\right)^{5-}$ elongates along the $c$-axis. Compared with $\mathrm{KBBF}$, the volumes of RBBF and CBBF expand by about $6 \%$ and $15 \%$, respectively. Table 1 also lists their NLO properties, demonstrating that the choice of $A$-site cations from $\mathrm{K}^{+}$to $\mathrm{Rb}^{+}$and $\mathrm{Cs}^{+}$does not affect the $E_{\mathrm{g}}$ and $d_{22}$, but reduces the $\Delta n=n_{\mathrm{o}}-n_{\mathrm{e}}$, because the $c$-axis $n_{\mathrm{e}}$ increases while the $a-b$ plane $n_{\mathrm{o}}$ keeps almost constant. This is so-called "NLO-size-effect" [56]. As such, the $\lambda_{\mathrm{PM}}$ of CBBF $>200 \mathrm{~nm}$ despite its $\lambda_{\mathrm{UV}} \approx 150 \mathrm{~nm}$, and the $d_{\text {eff }}$ of $\mathrm{RBBF}$ is only $0.5 \times \mathrm{KBBF}$ despite its $d_{22} \approx \mathrm{KBBF}$.

Depending on the "size-effect", the $A$-site substitute of $\mathrm{Na}^{+}$or $\mathrm{Li}^{+}$with smaller radius is good choice for DUV PM. $\mathrm{NaBe}_{2} \mathrm{BO}_{3} \mathrm{~F}_{2}$ (i.e., $\boldsymbol{\alpha}$-phase) is an existing compound with similar layering structural feature as KBBF but dif- ferent symmetry $C 2$ [59]. Fig. $2 \mathrm{~b}$ and Table 1 shows its structure and NLO properties. Due to the refractive dispersion caused by the sliding layers, the $\lambda_{\mathrm{PM}}$ is red-shifted by $13 \mathrm{~nm}$ than that of KBBF. It also shows a strong layering growth tendency. According to the $\mathrm{KBBF}$ structure, $\beta-\mathrm{NaBe}_{2} \mathrm{BO}_{3} \mathrm{~F}_{2}$ and $\mathrm{LiBe}_{2} \mathrm{BO}_{3} \mathrm{~F}_{2}$ with $R 32$ symmetry were further designed. The calculated $\Delta n$ are 0.064 and 0.067 , both larger than that of KBBF, and their $\lambda_{\mathrm{PM}}$ are blue-shifted by 2 and $7 \mathrm{~nm}$, respectively, due to the "size-effect"; however, phonon vibration analysis (Fig. S1) shows that they have instabilities owing to the presence of virtual frequencies. The interlayer slide of $\mathrm{Na}^{+} / \mathrm{Li}^{+}$makes the framework not stable.

If the improvement of single-crystal growth property is paid attention to, a possible approach is to use covalent $A$-site cations (e.g., $\mathrm{Tl}^{+}$or $\mathrm{Ag}^{+}$) instead of $\mathrm{K}^{+}, \mathrm{Rb}^{+}$or $\mathrm{Cs}^{+}$ to enhance the interlayer connection. $\mathrm{TlBe}_{2} \mathrm{BO}_{3} \mathrm{~F}_{2}$ is a representative with similar structure as $\mathrm{KBBF}$ and exhibits possibly better growth performance in preliminary experiments [60]. However, it has relatively small $E_{\mathrm{g}}$ $(\sim 6.3 \mathrm{eV})$ and $\Delta n(\sim 0.045)$, so it cannot achieve effective SHG output below $200 \mathrm{~nm}$. Another case is $\mathrm{AgBe}_{2} \mathrm{BO}_{3} \mathrm{~F}_{2}$, whose $E_{\mathrm{g}}$ is only $4.9 \mathrm{eV}$ (cal.) and which has not been DUV transparent. This means that the substitution with covalent $A$-site cations is not a good choice for DUV NLO crystals.

Since small-radius and covalent substitutes for the $A$ site cations hardly enhance DUV capability and single crystal growth property, an effective method is to use some relatively isolated ionic groups with certain covalence. By replacing $\mathrm{K}^{+}$in $\mathrm{KBBF}$ with ammonium ion
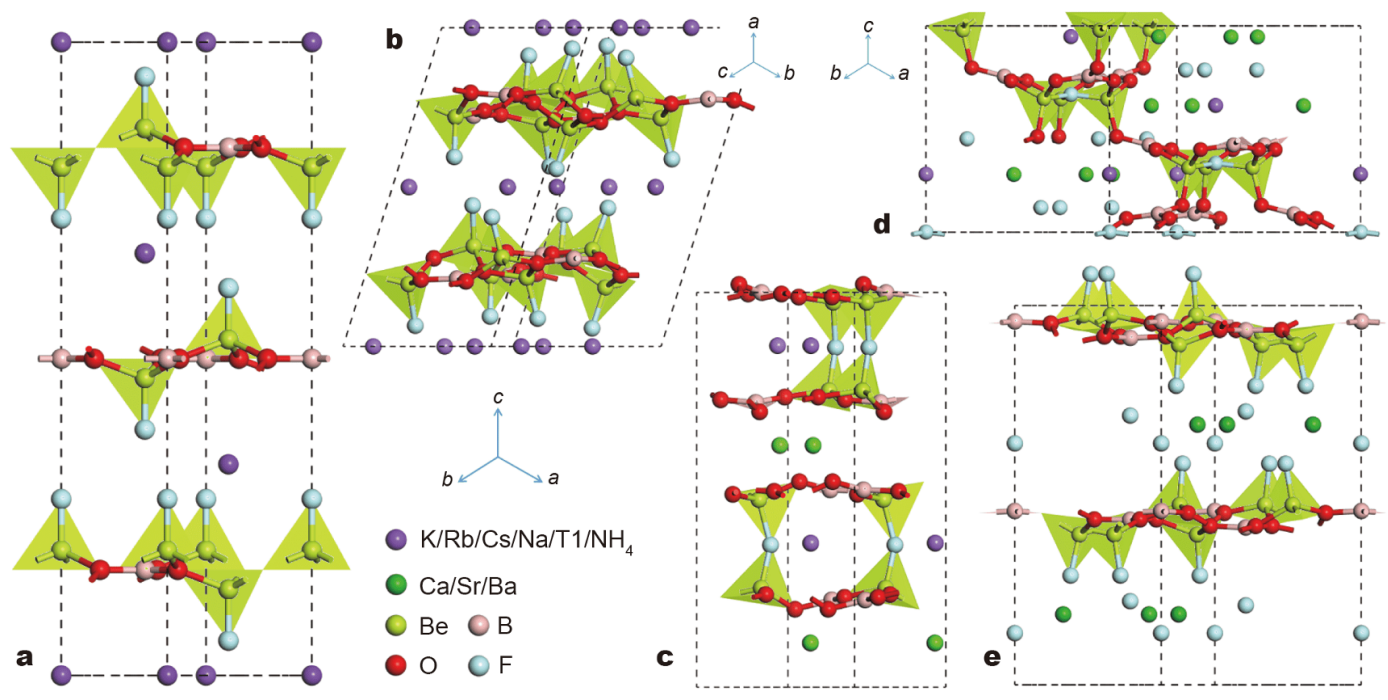

Figure 2 Structures of $A \mathrm{Be}_{2} \mathrm{BO}_{3} \mathrm{~F}_{2}\left(A=\mathrm{K}, \mathrm{Rb}, \mathrm{Cs}, \mathrm{Tl}, \mathrm{NH}_{4}\right)(\mathrm{a}), \mathrm{NaBe}_{2} \mathrm{BO}_{3} \mathrm{~F}_{2}(\mathrm{~b}), \mathrm{NaCaBe}_{2} \mathrm{~B}_{2} \mathrm{O}_{6} \mathrm{~F}$ (c), $\mathrm{NaSr}_{3} \mathrm{Be}_{3} \mathrm{~B}_{3} \mathrm{O}_{9} \mathrm{~F}_{4}(\mathrm{~d})$ and $\mathrm{BaBe}_{2} \mathrm{BO}_{3} \mathrm{~F}_{3}(\mathrm{e})$. 
Table 1 Calculated crystallographic data and NLO properties as compared to available experimental results

\begin{tabular}{|c|c|c|c|c|c|c|c|c|c|c|c|c|}
\hline & \multicolumn{2}{|c|}{ Symmetry } & $a(\AA)$ & $b(\AA)$ & $c(\AA)$ & $V\left(\AA^{3}\right)$ & $\begin{array}{c}E_{\mathrm{g}} \\
(\mathrm{eV})\end{array}$ & $\begin{array}{c}d_{i j} \\
\left(\mathrm{pm} \mathrm{V}^{-1}\right)\end{array}$ & $\begin{array}{c}\Delta n \text { at } \\
400 \mathrm{~nm}\end{array}$ & $\begin{array}{l}\lambda_{\mathrm{UV}} \\
(\mathrm{nm})\end{array}$ & $\begin{array}{l}\lambda_{\mathrm{PM}} \\
(\mathrm{nm})\end{array}$ & Refs \\
\hline \multicolumn{13}{|l|}{$A$-site substitute } \\
\hline \multirow[t]{2}{*}{$\mathrm{KBe}_{2} \mathrm{BO}_{3} \mathrm{~F}_{2}$} & Cal. & $R 32$ & 4.279 & 4.279 & 19.520 & 309.537 & 8.31 & $d_{22}=0.41$ & 0.059 & 150 & 172 & {$[56]$} \\
\hline & Exp. & $R 32$ & 4.427 & 4.427 & 18.744 & 318.135 & 8.45 & $d_{22}=0.47$ & 0.088 & 147 & 161 & {$[26]$} \\
\hline \multirow[t]{2}{*}{$\mathrm{RbBe}_{2} \mathrm{BO}_{3} \mathrm{~F}_{2}$} & Cal. & $R 32$ & 4.296 & 4.296 & 20.548 & 328.356 & 8.21 & $d_{22}=0.40$ & 0.057 & 151 & 185 & {$[56]$} \\
\hline & Exp. & $R 32$ & 4.439 & 4.439 & 19.820 & 338.178 & 8.18 & $d_{22}=0.45$ & 0.078 & 152 & 174 & [57] \\
\hline \multirow[t]{2}{*}{$\mathrm{CsBe}_{2} \mathrm{BO}_{3} \mathrm{~F}_{2}$} & Cal. & $R 32$ & 4.315 & 4.315 & 22.012 & 354.959 & 8.23 & $d_{22}=0.38$ & 0.055 & 151 & 210 & {$[56]$} \\
\hline & Exp. & $R 32$ & 4.458 & 4.458 & 21.310 & 366.688 & 8.23 & $d_{22}=0.50$ & 0.064 & 151 & 202 & {$[58]$} \\
\hline \multirow[t]{2}{*}{$\alpha-\mathrm{NaBe}_{2} \mathrm{BO}_{3} \mathrm{~F}_{2}$} & Cal. & $C 2$ & 7.723 & 7.723 & 7.341 & 372.459 & 8.26 & $d_{23}=0.44$ & 0.059 & 151 & 185 & This work \\
\hline & Exp. & $\mathrm{C} 2$ & 7.693 & 7.693 & 7.600 & 384.538 & 8.07 & $\sim \mathrm{KDP}$ & - & 154 & - & [59] \\
\hline$\beta-\mathrm{NaBe}_{2} \mathrm{BO}_{3} \mathrm{~F}_{2}^{\mathrm{a}}$ & Cal. & $R 32$ & 4.245 & 4.245 & 17.866 & 278.793 & 8.11 & $d_{22}=0.44$ & 0.064 & 153 & 170 & This work \\
\hline $\mathrm{LiBe}_{2} \mathrm{BO}_{3} \mathrm{~F}_{2}^{\mathrm{a}}$ & Cal. & $R 32$ & 4.228 & 4.228 & 17.023 & 263.537 & 8.38 & $d_{22}=0.46$ & 0.067 & 149 & 165 & This work \\
\hline \multirow[t]{2}{*}{$\mathrm{TlBe}_{2} \mathrm{BO}_{3} \mathrm{~F}_{2}$} & Cal. & $R 32$ & 4.290 & 4.290 & 20.640 & 328.950 & 6.58 & $d_{22}=0.46$ & 0.044 & 189 & 330 & This work \\
\hline & Exp. & $R 32$ & 4.439 & 4.439 & 19.942 & 340.260 & $\geq 6.30$ & $\sim \mathrm{KDP}$ & - & $\leq 200$ & - & {$[60]$} \\
\hline \multirow[t]{2}{*}{$\mathrm{NH}_{4} \mathrm{Be}_{2} \mathrm{BO}_{3} \mathrm{~F}_{2}$} & Cal. & $R 3$ & 4.299 & 4.299 & 20.658 & 330.642 & 8.37 & $\begin{array}{c}d_{22}=0.40 \\
d_{33}=0.23\end{array}$ & 0.053 & 148 & 188 & {$[36]$} \\
\hline & Exp. & $R 32$ & 4.442 & 4.442 & 19.909 & 340.170 & 8.12 & $\sim 1.2 \times \mathrm{KDP}$ & 0.091 & 153 & 174 & {$[37]$} \\
\hline $\mathrm{CH}_{3} \mathrm{NH}_{3} \mathrm{Be}_{2} \mathrm{BO}_{3} \mathrm{~F}_{2}^{\mathrm{a}}$ & Cal. & $R 3$ & 4.324 & 4.324 & 25.986 & 420.700 & 7.88 & $\begin{array}{l}d_{22}=0.30 \\
d_{33}=0.14\end{array}$ & 0.039 & 158 & 222 & This work \\
\hline \multirow{2}{*}{$\mathrm{NaCaBe}_{2} \mathrm{~B}_{2} \mathrm{O}_{6} \mathrm{~F}$} & Cal. & $C c$ & 4.536 & 4.536 & 14.257 & 254.065 & 7.21 & $d_{22}=0.18$ & 0.059 & 173 & 245 & {$[62]$} \\
\hline & Exp. & $C c$ & 4.623 & 4.623 & 14.182 & 262.527 & 6.52 & $\sim 0.5 \times \mathrm{KDP}$ & - & 191 & - & {$[62]$} \\
\hline \multirow[t]{2}{*}{$\mathrm{NaSr}_{3} \mathrm{Be}_{3} \mathrm{~B}_{3} \mathrm{O}_{9} \mathrm{~F}_{4}$} & Cal. & $R 3 m$ & 10.447 & 10.447 & 8.309 & 785.347 & 7.25 & $d_{12}=0.84$ & 0.048 & 172 & 248 & {$[63]$} \\
\hline & Exp. & $R 3 m$ & 10.406 & 10.406 & 8.289 & 777.367 & 7.29 & $\sim 2 \times \mathrm{KDP}$ & - & 171 & - & {$[63]$} \\
\hline \multirow[t]{2}{*}{$\mathrm{BaBe}_{2} \mathrm{BO}_{3} \mathrm{~F}_{3}$} & Cal. & $P 6_{3}$ & 7.628 & 7.628 & 13.990 & 704.950 & 8.41 & $d_{15}=0.02$ & 0.077 & 148 & 180 & {$[65]$} \\
\hline & Exp. & $P 6_{3}$ & 7.428 & 7.428 & 14.469 & 691.300 & $\geq 6.70$ & $\sim 0.3 \times \mathrm{KDP}$ & - & $\leq 186$ & - & {$[65]$} \\
\hline \multicolumn{13}{|l|}{$B$-site substitute } \\
\hline \multirow[t]{2}{*}{$\mathrm{BaAlBO}_{3} \mathrm{~F}_{2}$} & Cal. & $P \overline{6}$ & 4.814 & 4.814 & 9.390 & 188.474 & 7.61 & $d_{22}=0.70$ & 0.037 & 164 & 275 & {$[66]$} \\
\hline & Exp. & $P \overline{6}$ & 4.888 & 4.888 & 9.403 & 194.555 & 7.53 & $d_{22}=1.24$ & 0.042 & 165 & 273 & {$[66]$} \\
\hline \multirow[t]{2}{*}{$\mathrm{Rb}_{3} \mathrm{Al}_{3} \mathrm{~B}_{3} \mathrm{O}_{10} \mathrm{~F}$} & Cal. & $P 3_{1} c$ & 8.517 & 8.517 & 8.115 & 509.768 & 6.58 & $d_{22}=0.39$ & 0.063 & 189 & 235 & {$[67]$} \\
\hline & Exp. & $P 3_{1} c$ & 8.682 & 8.682 & 8.742 & 570.671 & $\geq 6.30$ & $\sim 1.2 \times \mathrm{KDP}$ & - & $\leq 200$ & - & {$[67]$} \\
\hline \multirow[t]{2}{*}{$\mathrm{K}_{3} \mathrm{Ba}_{3} \mathrm{Li}_{2} \mathrm{Al}_{4} \mathrm{~B}_{6} \mathrm{O}_{20} \mathrm{~F}$} & Cal. & $P \overline{6} 2 c$ & 8.584 & 8.584 & 16.343 & 1042.817 & 5.97 & $d_{22}=0.48$ & 0.053 & 209 & 248 & {$[68]$} \\
\hline & Exp. & $P \overline{6} 2 c$ & 8.755 & 8.755 & 16.435 & 1090.868 & 6.54 & $\sim 1.5 \times \mathrm{KDP}$ & - & 190 & - & {$[68]$} \\
\hline $\mathrm{KAlBO}_{3} \mathrm{~F}^{\mathrm{a}}$ & Cal. & $P \overline{6}$ & 5.016 & 5.016 & 3.744 & 81.594 & 7.15 & $\begin{array}{l}d_{11}=0.47 \\
d_{22}=0.66\end{array}$ & 0.078 & 174 & 182 & This work \\
\hline $\mathrm{KAl}_{2} \mathrm{~B}_{2} \mathrm{O}_{6} \mathrm{~F}^{\mathrm{a}}$ & Cal. & $R 32$ & 4.915 & 4.915 & 23.815 & 498.143 & 6.50 & $d_{22}=0.61$ & 0.091 & 191 & 192 & This work \\
\hline \multirow[t]{2}{*}{$\mathrm{BaZnBO}_{3} \mathrm{~F}$} & Cal. & $P \overline{6}$ & 4.880 & 4.880 & 4.561 & 94.089 & 5.69 & $\begin{array}{c}d_{11}=-0.67 \\
d_{22}=1.76\end{array}$ & 0.081 & 219 & 269 & [69] \\
\hline & Exp. & $P \overline{6}$ & 5.066 & 5.066 & 4.280 & 95.119 & 5.57 & $\sim 2.8 \times \mathrm{KDP}$ & - & 223 & - & [69] \\
\hline $\mathrm{KZn}_{2} \mathrm{BO}_{3} \mathrm{~F}_{2}^{\mathrm{a}}$ & Cal. & $R 32$ & 4.750 & 4.750 & 22.891 & 447.200 & 6.29 & $d_{22}=0.24$ & 0.046 & 197 & 266 & This work \\
\hline $\mathrm{KScBO}_{3} \mathrm{~F}^{\mathrm{a}}$ & Cal. & $P \overline{6}$ & 5.199 & 5.199 & 4.232 & 99.074 & 6.81 & $\begin{array}{l}d_{11}=2.03 \\
d_{22}=2.01\end{array}$ & 0.186 & 183 & 183 & This work \\
\hline $\mathrm{KYBO}_{3} \mathrm{~F}^{\mathrm{a}}$ & Cal. & $P \overline{6}$ & 5.014 & 5.014 & 4.489 & 97.757 & 6.11 & $\begin{array}{l}d_{11}=2.16 \\
d_{22}=1.02\end{array}$ & 0.179 & 203 & 204 & This work \\
\hline $\mathrm{KLaBO}_{3} \mathrm{~F}^{\mathrm{a}}$ & Cal. & $P \overline{6}$ & 4.789 & 4.789 & 4.390 & 87.185 & 5.91 & $\begin{array}{l}d_{11}=1.74 \\
d_{22}=0.00\end{array}$ & 0.183 & 211 & 210 & This work \\
\hline \multicolumn{13}{|l|}{$G$-site substitute } \\
\hline $\mathrm{KBe}_{2} \mathrm{~B}_{3} \mathrm{O}_{6} \mathrm{~F}_{2}{ }^{\mathrm{a}}$ & Cal. & $R 32$ & 6.152 & 6.152 & 13.672 & 448.077 & 7.44 & $d_{22}=0.83$ & 0.126 & 167 & 167 & This work \\
\hline $\mathrm{BaAlB}_{3} \mathrm{O}_{6} \mathrm{~F}_{2}{ }^{\mathrm{a}}$ & Cal. & $P \overline{6}$ & 6.475 & 6.475 & 7.787 & 282.752 & 7.86 & $d_{22}=1.01$ & 0.086 & 158 & 200 & This work \\
\hline
\end{tabular}




\begin{tabular}{|c|c|c|c|c|c|c|c|c|c|c|c|c|}
\hline \multirow[b]{2}{*}{$\mathrm{NH}_{4} \mathrm{~B}_{4} \mathrm{O}_{6} \mathrm{~F}$} & \multicolumn{2}{|c|}{ Symmetry } & \multirow{2}{*}{$\begin{array}{l}a(\AA) \\
8.450\end{array}$} & \multirow{2}{*}{$\begin{array}{c}b(\AA) \\
10.838\end{array}$} & \multirow{2}{*}{$\frac{c(\AA)}{6.495}$} & \multirow{2}{*}{$\begin{array}{c}V\left(\AA^{3}\right) \\
594.858\end{array}$} & \multirow{2}{*}{$\begin{array}{c}\begin{array}{c}E_{\mathrm{g}} \\
(\mathrm{eV})\end{array} \\
7.87\end{array}$} & \multirow{2}{*}{$\begin{array}{c}\begin{array}{c}d_{i j} \\
\left(\mathrm{pm} \mathrm{V}^{-1}\right)\end{array} \\
d_{32}=0.72 ; \\
d_{33}=-0.91\end{array}$} & \multirow{2}{*}{$\begin{array}{c}\begin{array}{c}\Delta n \text { at } \\
400 \mathrm{~nm}\end{array} \\
0.112\end{array}$} & \multirow{2}{*}{$\begin{array}{c}\begin{array}{l}\lambda_{\mathrm{UV}} \\
(\mathrm{nm})\end{array} \\
158\end{array}$} & \multirow{2}{*}{$\begin{array}{l}\begin{array}{l}\lambda_{\mathrm{PM}} \\
(\mathrm{nm})\end{array} \\
164\end{array}$} & \multirow{2}{*}{$\frac{\text { Refs }}{[40]}$} \\
\hline & Cal. & Pna2 $_{1}$ & & & & & & & & & & \\
\hline & Exp. & Pna2 $_{1}$ & 7.602 & 11.197 & 6.595 & 561.400 & 7.97 & $\sim 3 \times \mathrm{KDP}$ & 0.130 & 156 & 158 & [40] \\
\hline $\mathrm{BaB}_{4} \mathrm{O}_{6} \mathrm{~F}_{2}^{\mathrm{a}}$ & Cal. & $C c$ & 6.542 & 11.496 & 10.374 & 752.922 & 7.80 & $\begin{array}{c}d_{11}=-0.42 \\
d_{12}=0.57\end{array}$ & 0.065 & 160 & 180 & {$[74]$} \\
\hline $\mathrm{KB}_{2} \mathrm{O}_{3} \mathrm{~F}^{\mathrm{a}}$ & Cal. & $P 3$ & 4.360 & 4.360 & 4.976 & 81.911 & 7.46 & $\begin{aligned} d_{22} & =0.76 \\
d_{33} & =-0.55\end{aligned}$ & 0.064 & 167 & 180 & This work \\
\hline $\mathrm{KB}_{4} \mathrm{O}_{6} \mathrm{~F}^{\mathrm{a}}$ & Cal. & $R 32$ & 4.365 & 4.365 & 23.983 & 395.650 & 7.53 & $d_{22}=1.00$ & 0.083 & 165 & 176 & This work \\
\hline \multirow[t]{2}{*}{$\mathrm{KCaCO}_{3} \mathrm{~F}$} & Cal. & $P \overline{6} m 2$ & 5.097 & 5.097 & 4.455 & 100.231 & 6.31 & $d_{11}=1.33$ & 0.112 & 197 & 197 & [75] \\
\hline & Exp. & $P \overline{6} m 2$ & 5.063 & 5.063 & 4.576 & 101.577 & $\geq 6.30$ & $\sim 3.3 \times \mathrm{KDP}$ & - & $\leq 200$ & - & [35] \\
\hline $\mathrm{KBeCO}_{3} \mathrm{~F}^{\mathrm{a}}$ & Cal. & Amm2 & 5.444 & 5.444 & 2.970 & 83.875 & 7.61 & $\begin{array}{c}d_{11}=-0.70 \\
d_{12}=0.94\end{array}$ & 0.129 & 164 & 163 & [35] \\
\hline $\mathrm{KAlCO}_{3} \mathrm{~F}_{2}{ }^{\mathrm{a}}$ & Cal. & $P \overline{6} 2 c$ & 4.699 & 4.699 & 10.473 & 200.289 & 8.41 & $d_{22}=0.73$ & 0.108 & 148 & 147 & [35] \\
\hline $\mathrm{AlNO}_{3} \mathrm{~F}_{2}{ }^{\mathrm{a}}$ & Cal. & $P \overline{6} 2 c$ & 4.773 & 4.773 & 8.672 & 171.088 & 7.18 & $d_{22}=1.63$ & 0.178 & 174 & 174 & {$[77]$} \\
\hline $\mathrm{KSiBO}_{3} \mathrm{~F}_{2}^{\mathrm{a}}$ & Cal. & $P \overline{6}$ & 4.749 & 4.749 & 10.302 & 201.195 & 8.47 & $d_{16}=0.48$ & 0.060 & 147 & 178 & This work \\
\hline $\mathrm{KSiB}_{3} \mathrm{O}_{6} \mathrm{~F}_{2}{ }^{\mathrm{a}}$ & Cal. & $P \overline{6}$ & 6.820 & 6.820 & 8.663 & 348.944 & 7.75 & $d_{16}=0.53$ & 0.100 & 161 & 161 & This work \\
\hline $\mathrm{PBO}_{3} \mathrm{~F}_{2}^{\mathrm{a}}$ & Cal. & $P \overline{6} 2 c$ & 4.683 & 4.683 & 9.575 & 181.817 & 9.21 & $d_{16}=0.61$ & 0.076 & 135 & 155 & {$[81]$} \\
\hline $\mathrm{PB}_{3} \mathrm{O}_{6} \mathrm{~F}_{2}^{\mathrm{a}}$ & Cal. & $P \overline{6} 2 c$ & 6.862 & 6.862 & 9.089 & 370.619 & 9.10 & $d_{16}=0.45$ & 0.099 & 137 & 137 & {$[81]$} \\
\hline \multicolumn{13}{|l|}{$H$-site substitute } \\
\hline $\mathrm{KBe}_{2} \mathrm{BO}_{3} \mathrm{Cl}_{2}^{\mathrm{a}}$ & Cal. & $R 32$ & 4.304 & 4.304 & 26.162 & 419.682 & 7.63 & $d_{22}=0.39$ & 0.005 & 163 & N/A & This work \\
\hline $\mathrm{KBe}_{2} \mathrm{BO}_{3} \mathrm{H}_{2}{ }^{\mathrm{a}}$ & Cal. & $R 32$ & 4.264 & 4.264 & 16.863 & 265.562 & 6.70 & $d_{22}=0.59$ & 0.016 & 186 & N/A & This work \\
\hline $\mathrm{KBe}_{2} \mathrm{BO}_{3}(\mathrm{OH})_{2}{ }^{\mathrm{a}}$ & Cal. & $R 32$ & 4.321 & 4.321 & 21.269 & 343.903 & 6.63 & $d_{22}=0.46$ & 0.057 & 188 & 250 & This work \\
\hline \multicolumn{13}{|l|}{ Without $A$-site } \\
\hline \multirow[t]{2}{*}{$\alpha-\mathrm{Be}_{2} \mathrm{BO}_{3} \mathrm{~F}$} & Cal. & $C 2$ & 4.283 & 4.283 & 8.559 & 130.169 & 8.40 & $d_{15}=0.02$ & 0.077 & 148 & 180 & {$[82]$} \\
\hline & Exp. & $C 2$ & 4.438 & 4.438 & 8.699 & 141.870 & $\geq 6.70$ & $\sim 0.3 \times \mathrm{KDP}$ & - & $\leq 186$ & - & {$[82]$} \\
\hline \multirow[t]{2}{*}{$\gamma-\mathrm{Be}_{2} \mathrm{BO}_{3} \mathrm{~F}$} & Cal. & $R 32$ & 4.283 & 4.283 & 12.288 & 195.176 & 8.88 & $d_{12}=0.63$ & 0.094 & 140 & 149 & {$[36]$} \\
\hline & Exp. & $R 32$ & 4.440 & 4.440 & 12.470 & 212.870 & $\geq 6.30$ & $\sim 2.3 \times \mathrm{KDP}$ & - & $\leq 200$ & - & {$[37]$} \\
\hline $\mathrm{YCO}_{3} \mathrm{~F}^{\mathrm{a}}$ & Cal. & $P \overline{6} 2 c$ & 4.747 & 4.747 & 4.395 & 85.761 & 7.40 & $d_{11}=2.62$ & 0.197 & 168 & 168 & {$[77]$} \\
\hline $\mathrm{AlCO}_{3} \mathrm{~F}^{\mathrm{a}}$ & Cal. & $P \overline{6} 2 c$ & 4.793 & 4.793 & 3.414 & 67.920 & 8.51 & $d_{22}=0.98$ & 0.153 & 146 & 146 & {$[77]$} \\
\hline $\mathrm{Be}_{2} \mathrm{CO}_{3} \mathrm{~F}_{2}^{\mathrm{a}}$ & Cal. & $R 32$ & 4.215 & 4.215 & 14.180 & 218.167 & 8.46 & $d_{22}=0.87$ & 0.111 & 147 & 155 & {$[77]$} \\
\hline $\mathrm{SiCO}_{3} \mathrm{~F}_{2}{ }^{\mathrm{a}}$ & Cal. & $P \overline{6} 2 c$ & 4.658 & 4.658 & 9.434 & 177.261 & 9.64 & $d_{22}=0.96$ & 0.135 & 129 & 129 & [77] \\
\hline
\end{tabular}

a) represents that the compounds are theoretically designed.

$\left(\mathrm{NH}_{4}{ }^{+}\right)$, we directly obtained $\mathrm{NH}_{4} \mathrm{Be}_{2} \mathrm{BO}_{3} \mathrm{~F}_{2}$ (ABBF) with $R 3$ symmetry by selecting the same polar-axis of $\mathrm{NH}_{4}{ }^{+}$ [36]. Clearly, $\mathrm{ABBF}$ is analogous to $\mathrm{KBBF}$, and its size is between those of KBBF and RBBF (Table 1) because the radius of $\mathrm{NH}_{4}{ }^{+}$is between those of $\mathrm{K}^{+}$and $\mathrm{Rb}^{+}$. Based on the "NLO-size effect, ABBF"s $\Delta n$ is expected to be smaller than that of KBBF while larger than that of RBBF/CBBF.

Calculations show that ABBF has close $\lambda_{\mathrm{UV}}(\sim 150 \mathrm{~nm})$ and $d_{22}(\sim 1.2 \times \mathrm{KDP})$ to those of KBBF, RBBF and CBBF. However, the $\lambda_{\mathrm{PM}}$ is red-shifted from KBBF to ABBF, RBBF and CBBF. ABBF exhibits comparable DUV NLO properties with RBBF, demonstrating it could theoretically achieve the DUV $193.7 \mathrm{~nm}$-output like RBBF. The dynamical stability of $\mathrm{ABBF}$ was further verified by phonon calculations. The phonon spectra of ABBF and $\mathrm{KBBF}$ are similar, with no imaginary phonon mode observed (Fig. S1), indicating that $\mathrm{ABBF}$ is dynamically stable as KBBF. In fact, the compound has been obtained in recent experiments [37]. The calculated bulk and Young moduli revealed that ABBF has more favorable mechanical properties than KBBF, especially in the $c$-direction [36].

In short, $\mathrm{ABBF}$ is a newly obtained KBBF-family member using our proposed modeling system. In addition to $\mathrm{NH}_{4}{ }^{+}$, some other groups, e.g., $\mathrm{CH}_{3} \mathrm{NH}_{3}{ }^{+}, \mathrm{HC}\left(\mathrm{NH}_{2}\right)_{2}{ }^{+}$, $\mathrm{CH}_{3} \mathrm{C}\left(\mathrm{NH}_{2}\right)_{2}{ }^{+}, \mathrm{C}\left(\mathrm{NH}_{2}\right)_{3}{ }^{+},\left(\mathrm{CH}_{3}\right)_{3} \mathrm{NH}^{+}$, and $\left(\mathrm{CH}_{3}\right)_{2} \mathrm{C}(\mathrm{H})-$ 
$\mathrm{NH}_{3}{ }^{+}$, may be used as the $A$-site cationic candidates to form the $\mathrm{ABBF}$ analogues. For example, we have also designed $\mathrm{CH}_{3} \mathrm{NH}_{3} \mathrm{Be}_{2} \mathrm{BO}_{3} \mathrm{~F}_{2}$ (MABBF) to show the related NLO properties. The size of the MA is larger than that of $\mathrm{NH}_{4}{ }^{+}$especially along the $c$-axis, so its $\Delta n$ becomes smaller than that of ABBF. Table 1 lists its NLO properties, demonstrating that it cannot achieve the DUV output $\left(\lambda_{\mathrm{PM}}>200 \mathrm{~nm}\right)$ although its $E_{\mathrm{g}}$ is large enough $(\sim 7.9 \mathrm{eV})$.

$\mathrm{NaCaBe} e_{2} \mathrm{~B}_{2} \mathrm{O}_{6} \mathrm{~F}, \mathrm{NaSr}_{3} \mathrm{Be}_{3} \mathrm{~B}_{3} \mathrm{O}_{9} \mathrm{~F}_{4}$ and $\mathrm{BaBe}_{2} \mathrm{BO}_{3} \mathrm{~F}_{3}$ If some oxygen-bridged bonds are added instead of ionic interactions between layers, the growth property would be improved, which can be shown from the single-layered KBBF-type structures and double-layered $\mathrm{Sr}_{2} \mathrm{Be}_{2} \mathrm{~B}_{2} \mathrm{O}_{7}$-type structures [61]. However, the dangling orbitals of the $\mathrm{O}^{2-}$ bridge atoms reduce the $E_{\mathrm{g}}$. In order to improve the interlayer connection with few nonbonding states, using Fbridge bonds instead of $\mathrm{O}$-bridge bonds is considered as a good strategy, e.g., in $\mathrm{NaCaBe}_{2} \mathrm{~B}_{2} \mathrm{O}_{6} \mathrm{~F}$ and $\mathrm{NaSr}_{3} \mathrm{Be}_{3} \mathrm{~B}_{3} \mathrm{O}_{9} \mathrm{~F}_{4}$ $[62,63]$. For $\mathrm{NaCaBe}_{2} \mathrm{~B}_{2} \mathrm{O}_{6} \mathrm{~F}$, the SHG effect is only $0.5 \times \mathrm{KDP}$ because of the non-parallel alignment of $\left(\mathrm{BO}_{3}\right)^{3-}$ (Fig. 2c). For $\mathrm{NaSr}_{3} \mathrm{Be}_{3} \mathrm{~B}_{3} \mathrm{O}_{9} \mathrm{~F}_{4}$, the layer-structure declines to an umbrella shape but not an ideal plane, so $\Delta n$ becomes smaller than that of KBBF (Fig. 2d). This crystal cannot produce the DUV SHG output although it has strong SHG effect $(\sim 2 \times \mathrm{KDP})$ and large-size crystal $(\sim 13 \mathrm{~mm} \times 14 \mathrm{~mm} \times 20 \mathrm{~mm})$ [64].

In addition, using alkaline earth metals, e.g., $\mathrm{Ca}^{2+}, \mathrm{Sr}^{2+}$, $\mathrm{Ba}^{2+}$, as the $A$-site cations might enhance the interlayer interaction. $\mathrm{BaBe}_{2} \mathrm{BO}_{3} \mathrm{~F}_{3}(\mathrm{BBBF})$ is an example with layerstructures as shown in Fig. 2e [65]. Unfortunately, this structure exhibits too small SHG effect because of the anti-parallel arrangement of $\left(\mathrm{BO}_{3}\right)^{3-}$. If $\left(\mathrm{BO}_{3}\right)^{3-}$ can be aligned in a parallel pattern, $\mathrm{BBBF}$ would exhibit large SHG effect and achieve the DUV PM output.

From the above discussion, one may conclude that the $A$-site cationic substitute, one of the most important ways to enrich the KBBF-like system, can be used to modulate the NLO response of active anionic units for achieving the DUV NLO balance. In the practical design strategies, basically, $\mathrm{K}^{+}$is the first choice of $A$-site cations for $A_{i} B_{j} G_{m} H_{n}$-type compounds. Meanwhile, $\mathrm{NH}_{4}^{+}$can be a candidate for improving the interlayer connection. In addition, $\mathrm{Ba}^{2+}$ is also suitable to be the $A$-site cation when the charge should be balanced, which can still maintain an effective DUV NLO capability.

\section{$B$-site cationic substitute}

The $B$-site cations include $\mathrm{Be}^{2+}, \mathrm{Al}^{3+}, \mathrm{Zn}^{2+}, \mathrm{Sc}^{3+}$, etc., which are usually attached to the anionic groups to constitute the skeleton structures. The primary role of $B$ site cations in DUV NLO crystals is to saturate the nonbonding states and keep an anisotropic NLO-active structure.

\section{$\mathrm{BaAlBO}_{3} \mathrm{~F}_{2}, \mathrm{Rb}_{3} \mathrm{Al}_{3} \mathrm{~B}_{3} \mathrm{O}_{10} \mathrm{~F}$ and $\mathrm{KAlBO}_{3} \mathrm{~F}$}

$\mathrm{BaAlBO}_{3} \mathrm{~F}_{2}(\mathrm{BABF})$ is a typical NLO crystal with $E_{\mathrm{g}} \approx$ $7.5 \mathrm{eV}$ and $d_{22} \approx 3 \times \mathrm{KDP}$ [66]. Fig. 3a shows its KBBF-like structure composed by $\left(\mathrm{AlBO}_{3} \mathrm{~F}_{2}\right)_{\infty}$ layers. Due to the low structural anisotropy of $\left(\mathrm{AlO}_{3} \mathrm{~F}_{2}\right)^{5-}$, it has too small $\Delta n$ $(\sim 0.04)$ to satisfy the DUV PM requirement. Even if we introduce $\mathrm{Ca}^{2+}$ instead of $\mathrm{Ba}^{2+}$ to reduce the size according to the "size-effect", the $\Delta n$ just increases from 0.04 to 0.05 , still insufficiently large. Introducing alkalimetal cations instead of $\mathrm{Ba}^{2+}$ and interlayer F-bridged bond instead of $\mathrm{F}-\mathrm{Ba}$ ionic bond are feasible to enlarge $\Delta n$. This can be shown by experimental $\mathrm{Rb}_{3} \mathrm{Al}_{3} \mathrm{~B}_{3} \mathrm{O}_{10} \mathrm{~F}$ [67], which features a framework composed by $\left(\mathrm{Al}_{3}\left(\mathrm{BO}_{3}\right)_{3} \mathrm{OF}\right)_{\infty}$ layers and $\mathrm{Al}-\mathrm{F}$ bridged bonds (Fig. $3 \mathrm{~b}$ ). However, calculations show that $\Delta n \approx 0.06$ and $E_{\mathrm{g}} \approx$ $6.6 \mathrm{eV}$ are still not large enough, which result in inapplicable $\lambda_{\mathrm{PM}} \approx 235 \mathrm{~nm}$. Similar case includes another experimental $\mathrm{K}_{3} \mathrm{Ba}_{3} \mathrm{Li}_{2} \mathrm{Al}_{4} \mathrm{~B}_{6} \mathrm{O}_{20} \mathrm{~F}$ [68].

To enlarge the DUV $E_{\mathrm{g}}$ and $\Delta n$, we designed two structures, $\mathrm{KAlBO}_{3} \mathrm{~F}$ and $\mathrm{KAl}_{2} \mathrm{~B}_{2} \mathrm{O}_{6} \mathrm{~F}$, as shown in Fig. $3 \mathrm{C}$ and $\mathrm{d}$. Note that $\left(\mathrm{AlO}_{3} \mathrm{~F}_{2}\right)^{5-}$ bipyramids are connected by $\left(\mathrm{BO}_{3}\right)^{3-}$ triangles to form the $\left(\mathrm{Al}_{3} \mathrm{~B}_{3} \mathrm{O}_{6} \mathrm{~F}_{6}\right)$-rings, which are further linked by the apical $\mathrm{F}$ to form $3 \mathrm{D}$ framework. Such a structure makes these compounds exhibit larger $E_{\mathrm{g}}$ $(\sim 7.2$ and $6.5 \mathrm{eV})$ and $\Delta n(\sim 0.08$ and 0.09$)$ than those of $\mathrm{Rb}_{3} \mathrm{Al}_{3} \mathrm{~B}_{3} \mathrm{O}_{10} \mathrm{~F}$, so both can achieve the PM SHG output below $200 \mathrm{~nm}\left(\lambda_{\mathrm{PM}} \approx 182\right.$ and $\left.192 \mathrm{~nm}\right)$. Although they cannot output the practical 177.3-nm laser through 6HG, they are at least available for 193.7 nm-laser output. Moreover, they are dynamically stable from the analysis of phonon spectra (Fig. S1), indicating the synthesis possibility in the experiments.

$B a Z n B O_{3} F, K Z n_{2} B_{3} F_{2}$ and $K R E B O_{3} F(R E=S c, Y, L a)$ One can also consider $\mathrm{Zn}^{2+}$ as a possible $B$-site cation due to its chemical similarity as $\mathrm{Be}^{2+}$. $\mathrm{BaZnBO}_{3} \mathrm{~F}$ belongs to this NLO crystal with $E_{\mathrm{g}} \approx 5.6 \mathrm{eV}$ and $d_{\mathrm{ij}} \approx 3 \times \mathrm{KDP}[69]$. Obviously, its $E_{\mathrm{g}}$ becomes too small to be transparent to DUV light due to relatively weak $\mathrm{Zn}-\mathrm{O}$ coupling. For $\mathrm{KZn}_{2} \mathrm{BO}_{3} \mathrm{~F}_{2}$ with designed KBBF-like structure, its $E_{\mathrm{g}} \approx$ $6.3 \mathrm{eV}$ is also not suitable. Basically, small $E_{\mathrm{g}}$ is the reason why Zn-based KBBF-like structures cannot be applied as DUV NLO materials.

As an alternative to $B$-site cation, rare-earth-metalbased compounds greatly enrich the NLO borate field, 


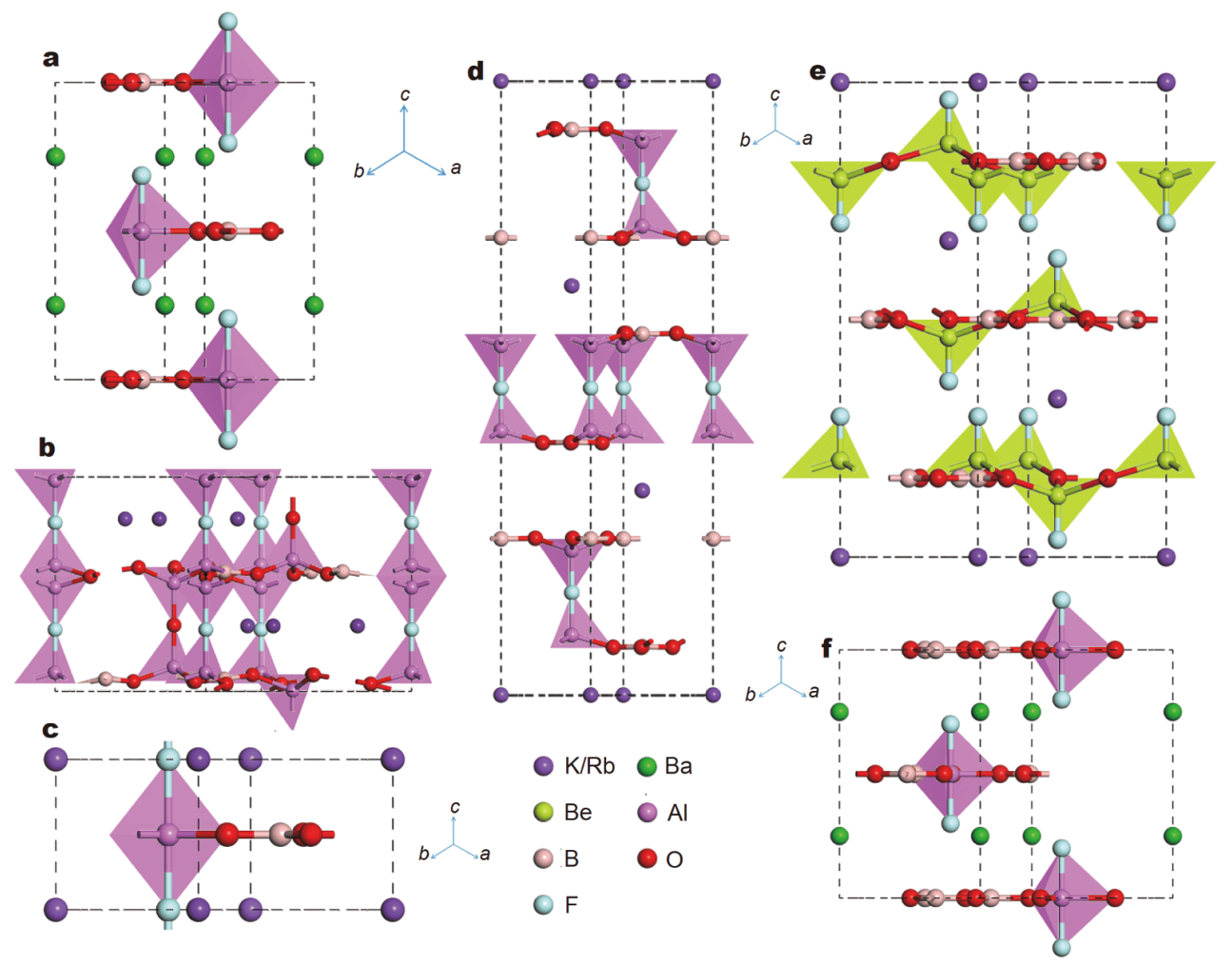

Figure 3 Structures of $\mathrm{BaAlBO}_{3} \mathrm{~F}_{2}(\mathrm{a}), \mathrm{Rb}_{3} \mathrm{Al}_{3} \mathrm{~B}_{3} \mathrm{O}_{10} \mathrm{~F}(\mathrm{~b}), \mathrm{KAlBO}_{3} \mathrm{~F}(\mathrm{c}), \mathrm{KAl}_{2} \mathrm{~B}_{2} \mathrm{O}_{6} \mathrm{~F}$ (d), $\mathrm{KBe}_{2} \mathrm{~B}_{3} \mathrm{O}_{6} \mathrm{~F}_{2}$ (e) and $\mathrm{BaAlB}_{3} \mathrm{O}_{6} \mathrm{~F}_{2}$ (f).

including $\mathrm{YAl}_{3}\left(\mathrm{BO}_{3}\right)_{4}[31], \mathrm{LaBeB}_{3} \mathrm{O}_{7}[70], R E \mathrm{Be}_{2} \mathrm{~B}_{5} \mathrm{O}_{11}$ $(R E=\mathrm{Y}, \mathrm{Gd})$ [71], which exhibit potential DUV NLO capabilities. The $R E$-site atoms usually include $\mathrm{Sc}^{3+}, \mathrm{Y}^{3+}$ and $\mathrm{La}^{3+}$. Accordingly, we designed a series of KBBF-like $R E$-based borates of $\mathrm{KREBO}_{3} \mathrm{~F}(R E=\mathrm{Sc}, \mathrm{Y}, \mathrm{La})$ similar to $\mathrm{KAlBO}_{3} \mathrm{~F}$. Their NLO properties show that only $\mathrm{KScBO}_{3} \mathrm{~F}$ can achieve the DUV output whereas $\mathrm{KYBO}_{3} \mathrm{~F}$ and $\mathrm{KLaBO}_{3} \mathrm{~F}$ cannot because of their relatively small $E_{\mathrm{g}}$. Note that $\mathrm{KScBO}_{3} \mathrm{~F}$ exhibits much larger SHG effect $(\sim 5 \times \mathrm{KDP})$ than most of the DUV materials $(1-3 \times \mathrm{KDP})$, so it can achieve much higher conversion efficiency, which is an advantage of this NLO class.

From the discussion of this section, the $B$-site cationic substitute is an effective way for balancing $E_{\mathrm{g}}$ and $\Delta n$. $\mathrm{Al}^{3+}$ and $\mathrm{Sc}^{3+}$ can be used as two $B$-site candidates. If we choose $\mathrm{NH}_{4}{ }^{+}$(or $\mathrm{Li}^{+}$) instead of $\mathrm{K}^{+}$in $\mathrm{KAlBO}_{3} \mathrm{~F}, \mathrm{KAl}_{2} \mathrm{~B}_{2} \mathrm{O}_{6} \mathrm{~F}$ or $\mathrm{KREBO}_{3} \mathrm{~F}$, the DUV NLO properties would be tunable and well maintained based on the NLO size-effect.

\section{$G$-site anionic group substitute}

Anionic group theory tells us covalent borate anionic groups play a dominant role in the NLO responses. There are four main anionic groups in borates, i.e., $\left(\mathrm{B}_{3} \mathrm{O}_{6}\right)^{3-}$ (e.g., in $\mathrm{BBO}),\left(\mathrm{B}_{3} \mathrm{O}_{7}\right)^{5-}$ (e.g., in $\left.\mathrm{LBO}\right),\left(\mathrm{BO}_{3}\right)^{3-}$ (e.g., in
$\mathrm{KBBF}$ ) and $\left(\mathrm{BO}_{4}\right)^{5-}$ (e.g., in $\left.\mathrm{SrB}_{4} \mathrm{O}_{7}\right)$ [72], which are considered as four NLO material genomes (of course, it is also reasonable to consider that $\mathrm{BO}_{3}$ and $\mathrm{BO}_{4}$ are two basic units in borates, while $\mathrm{B}_{3} \mathrm{O}_{6}$ and $\mathrm{B}_{3} \mathrm{O}_{7}$ or $\mathrm{B}_{2} \mathrm{O}_{5}$, $\mathrm{B}_{5} \mathrm{O}_{10}$, etc. are composite building blocks), exhibiting exciting structural chemistry with a great variety of connection and arrangement patterns [73].

$\mathrm{KBe}_{2} \mathrm{~B}_{3} \mathrm{O}_{6} \mathrm{~F}_{2}, \mathrm{BaAlB}_{3} \mathrm{O}_{6} \mathrm{~F}_{2}, \mathrm{NH}_{4} \mathrm{~B}_{4} \mathrm{O}_{6} \mathrm{~F}$ and $\mathrm{BaB}_{4} \mathrm{O}_{6} \mathrm{~F}_{2}$ As an important NLO unit, $\left(\mathrm{B}_{3} \mathrm{O}_{6}\right)^{3-}$ in $\mathrm{BBO}$ exhibits wide $E_{\mathrm{g}}(\sim 6.7 \mathrm{eV})$, strong $d_{i j}(\sim 2 \times \mathrm{KDP})$ and large $\Delta n(\sim 0.10)$. Although it seems to achieve the DUV NLO balance, it cannot be used for DUV due to its unavailable $\lambda_{\mathrm{PM}} \approx$ $204 \mathrm{~nm}$, mainly originating from relatively small $E_{\mathrm{g}}$ [25]. Note that the refractive dispersion of BBO is not as good as that of $\mathrm{KBBF}$, which is another important reason why its PM ability is lower than that of KBBF. If the nonbonding states could be removed by coordinating covalent elements such as $\mathrm{Be}^{2+}$ or $\mathrm{Al}^{3+}$ with $\left(\mathrm{B}_{3} \mathrm{O}_{6}\right)^{3-}$, the system may become a DUV NLO material. Accordingly, based on $\mathrm{KBBF}$ and $\mathrm{BABF}$, we designed two compounds $\mathrm{KBe}_{2} \mathrm{~B}_{3} \mathrm{O}_{6} \mathrm{~F}_{2}$ and $\mathrm{BaAlB}_{3} \mathrm{O}_{6} \mathrm{~F}_{2}$, respectively, using $\left(\mathrm{B}_{3} \mathrm{O}_{6}\right)^{3-}$ instead of $\left(\mathrm{BO}_{3}\right)^{3-}$ and $\mathrm{Be}^{2+} / \mathrm{Al}^{3+}$ as the $B$-site cations. Fig. $3 e, f$ display their structures, in which the sizes within 
the $a-b$ plane are expanded by about $50 \%$ and squeezed by about $30 \%$ along the $c$-axis. As a result, the structural anisotropy is greatly enlarged from $\mathrm{KBBF}$ to $\mathrm{KBe}_{2} \mathrm{~B}_{3} \mathrm{O}_{6} \mathrm{~F}_{2}$ and $\mathrm{BaAlBO}_{3} \mathrm{~F}_{2}$ to $\mathrm{BaAlB}_{3} \mathrm{O}_{6} \mathrm{~F}_{2}$, making their $\Delta n$ and SHG effect greatly enhanced. Table 1 shows that $\Delta n$ and $d_{22}\left(0.12\right.$ and $0.83 \mathrm{pm} \mathrm{V}^{-1}$ for $\mathrm{KBe}_{2} \mathrm{~B}_{3} \mathrm{O}_{6} \mathrm{~F}_{2} ; 0.086$ and $1.01 \mathrm{pm} \mathrm{V}^{-1}$ for $\left.\mathrm{BaAlB}_{3} \mathrm{O}_{6} \mathrm{~F}_{2}\right)$ are much larger than those of $\mathrm{KBBF}\left(0.059\right.$ and $\left.0.41 \mathrm{pm} \mathrm{V}^{-1}\right)$ and BABF (0.037 and $0.70 \mathrm{pm} \mathrm{V}^{-1}$ ). Thus, their $\lambda_{\mathrm{PM}}$ are blue-shifted from 172 to $167 \mathrm{~nm}$ and 235 to $200 \mathrm{~nm}$.

Unfortunately, they are not dynamically stable due to the heavy virtual frequencies in phonon spectra (Fig. S1), which are mainly caused by the vibration modes from $\mathrm{BeO}_{3} \mathrm{~F}-\mathrm{B}_{3} \mathrm{O}_{6}$ and $\mathrm{AlO}_{3} \mathrm{~F}_{2}-\mathrm{B}_{3} \mathrm{O}_{6}$ groups. It is why similar structures are rare in ICSD. If we introduce $\left(\mathrm{BO}_{3} \mathrm{~F}\right)^{4-}$ to replace $\left(\mathrm{BeO}_{3} \mathrm{~F}\right)^{5-}$ in the structure to form $\left(\mathrm{B}_{4} \mathrm{O}_{7} \mathrm{~F}\right)^{-}$ group, the instabilities may be eliminated and the DUV capability would be improved. A typical example from recent experiments is $\mathrm{NH}_{4} \mathrm{~B}_{4} \mathrm{O}_{6} \mathrm{~F}(\mathrm{ABF})$ with a wave-like layer-framework linked by $\mathrm{NH}_{4}{ }^{+}$as shown in Fig. 4a [40]. Its NLO properties are excellent, including $E_{\mathrm{g}} \approx 8.0 \mathrm{eV}, d_{i j}$ $\approx 3 \times \mathrm{KDP}, \Delta n \approx 0.13$ and $\lambda_{\mathrm{PM}} \approx 158 \mathrm{~nm}$. Another example is $\mathrm{BaB}_{4} \mathrm{O}_{6} \mathrm{~F}_{2}$, which is designed theoretically and can achieve the DUV PM output of $180 \mathrm{~nm}$ [74]. Herein, we have also designed two novel structures, i.e., $\mathrm{KB}_{2} \mathrm{O}_{3} \mathrm{~F}$ and $\mathrm{KB}_{4} \mathrm{O}_{6} \mathrm{~F}$, with single-layer structure and double-layer structure as shown in Fig. $4 \mathrm{~b}$, c. Table 1 shows that they exhibit large $E_{\mathrm{g}} \approx 7.5 \mathrm{eV}, d_{22} \approx 2-2.5 \times \mathrm{KDP}$ and $\Delta n \approx$ $0.06-0.08$, so they can achieve DUV PM output of 180 and $176 \mathrm{~nm}$. Once obtained in experiments, they could be applied for the $193.7 \mathrm{~nm} / 177.3 \mathrm{~nm}$ laser.

$\mathrm{KCaCO}_{3} \mathrm{~F}, \mathrm{KBeCO}_{3} \mathrm{~F}, \mathrm{KAlCO}_{3} \mathrm{~F}_{2}$ and $\mathrm{AlNO}_{3} \mathrm{~F}_{2}$ Similar to $\left(\mathrm{BO}_{3}\right)^{3-},\left(\mathrm{CO}_{3}\right)^{2-}$ and $\left(\mathrm{NO}_{3}\right)^{-}$units exhibit planar triangle structures, so they are good units beneficial to large $d_{i j}$ and $\Delta n . \mathrm{KCaCO}_{3} \mathrm{~F}$ is a typical NLO carbonate with strong $d_{11}(\sim 3 \times \mathrm{KDP})$ and $\Delta n(\sim 0.11)$ but small $E_{\mathrm{g}}$ $(\sim 6.3 \mathrm{eV})$ because $\mathrm{Ca}^{2+}$ cannot remove the nonbonding states of $\left(\mathrm{CO}_{3}\right)^{2-}$ (Fig. 4d) [75]. Similar cases include other $A B \mathrm{CO}_{3}$ F-type $(A=\mathrm{K}, \mathrm{Rb}, \mathrm{Cs} ; B=\mathrm{Mg}, \mathrm{Ca}, \mathrm{Sr}$ ) carbonates [76]. For improving $E_{\mathrm{g}}$ while keeping $d_{i j}$ and $\Delta n$ suitable, we found an existing compound $\mathrm{Sr}_{2} \mathrm{AlCO}_{3} \mathrm{~F}_{5}$ exhibiting larger $E_{\mathrm{g}}(\sim 7.8 \mathrm{eV})$ than $\mathrm{KCaCO}_{3} \mathrm{~F}$ because $\mathrm{Al}^{3+}$ partially removed the nonbonding states of $\left(\mathrm{CO}_{3}\right)^{2-}$. Accordingly, we designed two series of $A \mathrm{BeCO}_{3} \mathrm{~F}$ and $A \mathrm{AlCO}_{3} \mathrm{~F}_{2}(A=$ $\mathrm{Li}, \mathrm{Na}, \mathrm{K}, \mathrm{Rb}, \mathrm{Cs}$ ) to evaluate the possible DUV NLO capabilities [35]. $\mathrm{KBeCO}_{3} \mathrm{~F}$ and $\mathrm{KAlCO}_{3} \mathrm{~F}_{2}$ as two representatives (Fig. 4e, f), have achieved the balance with $E_{\mathrm{g}}$ $\approx 7.6$ and $8.4 \mathrm{eV}$ and $\Delta n \approx 0.13$ and 0.11 , so they can output the DUV PM cutoff wavelength of 163 and $147 \mathrm{~nm}$. Moreover, their SHG effects are larger than that of KBBF. As dynamically stable phases, $A \mathrm{AlCO}_{3} \mathrm{~F}_{2}$ were the first series predicted to break the limit of $\mathrm{KBBF}$ with superior PM capabilities, although they have not been obtained in the follow-up experiments.

$\mathrm{AlNO}_{3} \mathrm{~F}_{2}$ is a typical NLO nitrate (Fig. 4g) with the balance of $E_{\mathrm{g}} \approx 7.0 \mathrm{eV}$ and $\Delta n \approx 0.22$ [77]. It can output a $178 \mathrm{~nm}$-output theoretically. It is the first possible nitrate that we have predicted as a DUV NLO compound. Similar to $\left(\mathrm{B}_{3} \mathrm{O}_{6}\right)^{3-}$, there is additionally a $\left(\mathrm{C}_{3} \mathrm{~N}_{3} \mathrm{O}_{3}\right)^{3-}$ unit that exhibits similar structure and NLO properties as $\mathrm{BBO}$ [78]. For example, $\mathrm{Ca}_{3}\left(\mathrm{C}_{3} \mathrm{~N}_{3} \mathrm{O}_{3}\right)_{2}$ has large SHG effect $\left(\sim 3.46 \mathrm{pm} \mathrm{V}^{-1}\right)$ and $\Delta n(\sim 0.35)$ but small $E_{\mathrm{g}}$ $(\sim 5.6 \mathrm{eV})$. If its nonbonding states can be eliminated as discussed in the case of $\left(\mathrm{B}_{3} \mathrm{O}_{6}\right)^{3-}$, then it could be used as possible DUV material.

$\mathrm{KSiBO}_{3} \mathrm{~F}_{2}, \mathrm{KSiB}_{3} \mathrm{O}_{6} \mathrm{~F}_{2}, \mathrm{PBO}_{3} \mathrm{~F}_{2}$ and $\mathrm{PB}_{3} \mathrm{O}_{6} \mathrm{~F}_{2}$

In general, the $B$-site elements e.g., $\mathrm{Be}, \mathrm{Al}, \mathrm{Sc}$, exhibit relatively weaker covalence than $\mathrm{B}$, and tend to form the

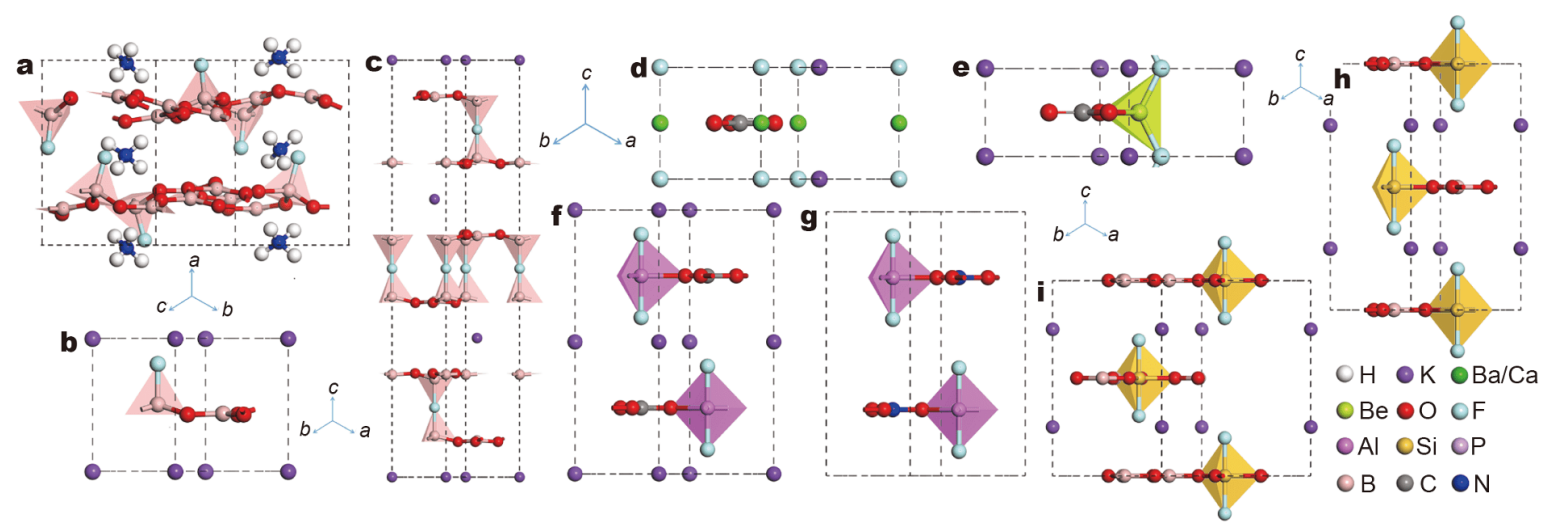

Figure 4 Structures of $\mathrm{NH}_{4} \mathrm{~B}_{4} \mathrm{O}_{6} \mathrm{~F}(\mathrm{a}), \mathrm{KB}_{2} \mathrm{O}_{3} \mathrm{~F}(\mathrm{~b}), \mathrm{KB}_{4} \mathrm{O}_{6} \mathrm{~F}(\mathrm{c}), \mathrm{KCaCO}_{3} \mathrm{~F}(\mathrm{~d}), \mathrm{KBeCO}_{3} \mathrm{~F}(\mathrm{e}), \mathrm{KAlCO}_{3} \mathrm{~F}_{2}(\mathrm{f}), \mathrm{AlNO}_{3} \mathrm{~F}_{2}(\mathrm{~g}), \mathrm{KSiBO}_{3} \mathrm{~F}_{2}(\mathrm{~h})$ and $\mathrm{KSiB}_{3} \mathrm{O}_{6} \mathrm{~F}_{2}(\mathrm{i})$. 
coordination bonds with borate groups. As the increase of covalence, the $B$-site elements will share more electrons with adjacent oxygen atoms to form the covalent bonds. Then these elements are no longer general $B$-site whereas should be defined as $G$-site. Such elements mainly include $\mathrm{C}, \mathrm{N}, \mathrm{Si}$ and P. According to Pauling rules of the chemical formation, $\mathrm{C}$ and $\mathrm{N}$ are difficult to coordinate with borate groups, so there are almost no known borocarbonates or boronitrates found in the experiments. However, once the covalent G-site elements (e.g., Si or P) are coordinated with the borate units, they would remove much more nonbonding states than the coordinated $B$-site elements (e.g., Be or Al). Therefore, it is necessary to evaluate the DUV NLO properties of Si-based and P-based systems since borosilicates and borophosphates are relatively abundant in ICSD. Unfortunately, most of the known borosilicates and borophosphates exhibit small structural anisotropy, so the $\Delta n$ is not sufficiently large. This is mainly because $\mathrm{Si}^{4+}$ and $\mathrm{P}^{5+}$ tend to form tetrahedral units such as $\left(\mathrm{SiO}_{4}\right)^{4-}$ and $\left(\mathrm{PO}_{4}\right)^{3-}$, which usually share the vertex-oxygen atoms to form $3 \mathrm{D}$ framework structures with low spatial anisotropy.

$\mathrm{BPO}_{4}$ is a typical borophosphate with tetrahedral $\left(\mathrm{BO}_{4}\right)^{5-}$ and $\left(\mathrm{PO}_{4}\right)^{3-}$ [79]. It has strong SHG effect $\left(\sim 0.76 \mathrm{pm} \mathrm{V}^{-1}\right)$ and extremely short DUV edge $(\sim 134 \mathrm{~nm})$. However, due to its small $\Delta n(<0.01), \mathrm{BPO}_{4}$ cannot produce the UV PM output. A similar example in borosilicates is $\mathrm{LiBSiO}_{4}$ with tetrahedral $\left(\mathrm{BO}_{4}\right)^{5-}$ and $\left(\mathrm{SiO}_{4}\right)^{4-}$ [80]. It also exhibits strong SHG effect $\left(\sim 0.70 \mathrm{pm} \mathrm{V}^{-1}\right)$ and wide $E_{\mathrm{g}}(\sim 8.8 \mathrm{eV})$ but small $\Delta n$ $(<0.01)$, and it cannot satisfy the DUV NLO requirements. If $\mathrm{BPO}_{4}$ and $\mathrm{LiBSiO}_{4}$ are expected as DUV NLO materials, they need to expand the structural anisotropy. To achieve this goal, the coplanar $\left(\mathrm{BO}_{3}\right)^{3-}$ or $\left(\mathrm{B}_{3} \mathrm{O}_{6}\right)^{3-}$ units are introduced to replace $\left(\mathrm{BO}_{4}\right)^{5-}$ and to coordinate with $\left(\mathrm{PO}_{4}\right)^{3-}$ in $\mathrm{BPO}_{4}$ or $\left(\mathrm{SiO}_{4}\right)^{5-}$ in $\mathrm{LiBSiO}_{4}$, thus to form a layered structural topology, which is favorable to exhibiting large $E_{\mathrm{g}}$ since the terminal $\mathrm{O}^{2-}$ of $\left(\mathrm{BO}_{3}\right)^{3-}$ or $\left(\mathrm{B}_{3} \mathrm{O}_{6}\right)^{3-}$ are bonded by $\mathrm{P}$ or $\mathrm{Si}$. Meanwhile, new structural units such as $\left(\mathrm{PO}_{m} \mathrm{~F}_{n}\right)$ or $\left(\mathrm{SiO}_{m} \mathrm{~F}_{n}\right)$ can be formed by incorporating fluorine into the lattice, which can further remove the dangling bonds and tune the pattern of anionic groups.

Accordingly, $\mathrm{KSiBO}_{3} \mathrm{~F}_{2}, \quad \mathrm{KSiB}_{3} \mathrm{O}_{6} \mathrm{~F}_{2}, \quad \mathrm{PBO}_{3} \mathrm{~F}_{2}$ and $\mathrm{PB}_{3} \mathrm{O}_{6} \mathrm{~F}_{2}$ were designed as the representative $\mathrm{KBBF}$-like borosilicates and borophosphates (Figs $4 \mathrm{~h}$, i and $5 \mathrm{a}, \mathrm{b}$ ) [81]. Since the nonbonding states are eliminated, $\mathrm{KSiBO}_{3} \mathrm{~F}_{2}, \mathrm{KSiB}_{3} \mathrm{O}_{6} \mathrm{~F}_{2}, \mathrm{PBO}_{3} \mathrm{~F}_{2}$ and $\mathrm{PB}_{3} \mathrm{O}_{6} \mathrm{~F}_{2}$ can be transparent in the DUV region. Table 1 lists their calculated $E_{\mathrm{g}}, d_{i j}, \Delta n$ and $\lambda_{\mathrm{PM}}$. Compared with $\mathrm{LiBSiO}_{4}$,
$\mathrm{KSiBO}_{3} \mathrm{~F}_{2}$ and $\mathrm{KSiB}_{3} \mathrm{O}_{6} \mathrm{~F}_{2}$ exhibit slightly smaller $E_{\mathrm{g}}(\sim 8.5$ and $7.7 \mathrm{eV})$, weaker $d_{i j}\left(\sim 0.48\right.$ and $\left.0.53 \mathrm{pm} \mathrm{V}^{-1}\right)$, larger $\Delta n$ ( $\sim 0.06$ and 0.10$)$ and shorter $\lambda_{\mathrm{PM}}(\sim 178$ and $161 \mathrm{~nm})$, thus exhibiting superior DUV PM abilities. Compared with $\mathrm{BPO}_{4}, \mathrm{PBO}_{3} \mathrm{~F}_{2}$ and $\mathrm{PB}_{3} \mathrm{O}_{6} \mathrm{~F}_{2}$ have comparable $E_{\mathrm{g}}$ ( 9.2 and $9.1 \mathrm{eV})$, weaker $d_{i j}\left(\sim 0.61\right.$ and $\left.0.45 \mathrm{pm} \mathrm{V}^{-1}\right)$, larger $\Delta n$ $(\sim 0.08$ and 0.10$)$ and shorter $\lambda_{\mathrm{PM}}(\sim 155$ and $137 \mathrm{~nm})$, also exhibiting superior DUV PM abilities.

From the discussion of this section, the $G$-site substitute is important to expand the systems with potential DUV NLO properties. In general, $\left(\mathrm{BO}_{3}\right)^{3-}$ exhibits a relatively large $E_{\mathrm{g}}$ but small $\Delta n,\left(\mathrm{NO}_{3}\right)^{-}$exhibits a relatively large $\Delta n$ but small $E_{\mathrm{g}}$, and $\left(\mathrm{CO}_{3}\right)^{2-}$ can achieve large $E_{\mathrm{g}}$ and $\Delta n$. However, borate-based anionic groups are more favorable to form some complex framework groups with other groups such as borate, silicate and phosphate groups than nitride or carbonate groups, which still play a dominant role in the DUV NLO field.

\section{$\boldsymbol{H}$-site anionic substitute}

The $H$-site anions are important for DUV NLO structures, which can eliminate the nonbonding states, adjust the NLO structure and balance the electronic charge. As the most effective $H$-site choice, fluoride possesses larger electronegativity and smaller atomic radius than oxygen. Therefore, fluoride oxides usually exhibit larger $E_{\mathrm{g}}$ than other kinds of oxides.

In addition, $\mathrm{F}$ can be introduced into some basic anionic units such as $\left(\mathrm{BeO}_{4}\right)^{6-}$ or $\left(\mathrm{BO}_{4}\right)^{5-}$ tetrahedral groups to form $\left(\mathrm{BeO}_{3} \mathrm{~F}\right)^{5-}$ or $\left(\mathrm{BO}_{3} \mathrm{~F}\right)^{4-}$ quasi-plane groups. As a result, the fluoride-oxide groups may exhibit relatively large structural anisotropy for large $\Delta n$. If $\mathrm{F}$ is replaced by other $\mathrm{H}$-site anions such as $\mathrm{Cl}^{-}, \mathrm{H}^{-}$, and $\mathrm{OH}^{-}$, the resulting $\mathrm{KBe}_{2} \mathrm{BO}_{3} \mathrm{X}_{2}(\mathrm{X}=\mathrm{Cl}, \mathrm{H}, \mathrm{OH})$ may not exhibit available DUV NLO properties as shown in Table 1.

\section{Without $A$-site cations}

As an important supplement of $A$-site substitute, reducing the $A$-site cationic size to zero will further enhance the DUV NLO properties in the KBBF system based on the "NLO size-effect". If some kinds of $2 \mathrm{D}$ layers can be formed by introduing the planar anonic units (e.g., $\left(\mathrm{BO}_{3}\right)^{3-}$ or $\left.\left(\mathrm{CO}_{3}\right)^{3-}\right)$, the structural anisotropy will be enlarged and the DUV PM conditions are possibly satisfied, which can be successfully shown in the following cases from $\alpha-\mathrm{Be}_{2} \mathrm{BO}_{3} \mathrm{~F}$ in Fig. $5 c$ to $\gamma-\mathrm{Be}_{2} \mathrm{BO}_{3} \mathrm{~F}$ in Fig. $5 d$.

\section{Bridge-bonded layer-structures and $\mathrm{Be}_{2} \mathrm{BO}_{3} \mathrm{~F}$}

$\mathrm{Be}_{2} \mathrm{BO}_{3} \mathrm{~F}$ has an existing $\alpha$-phase derived from $\mathrm{KBBF}$ by removing one $\mathrm{K}^{+}$and $\mathrm{F}^{-}$. It has large $\Delta n(\sim 0.08)$ and short 

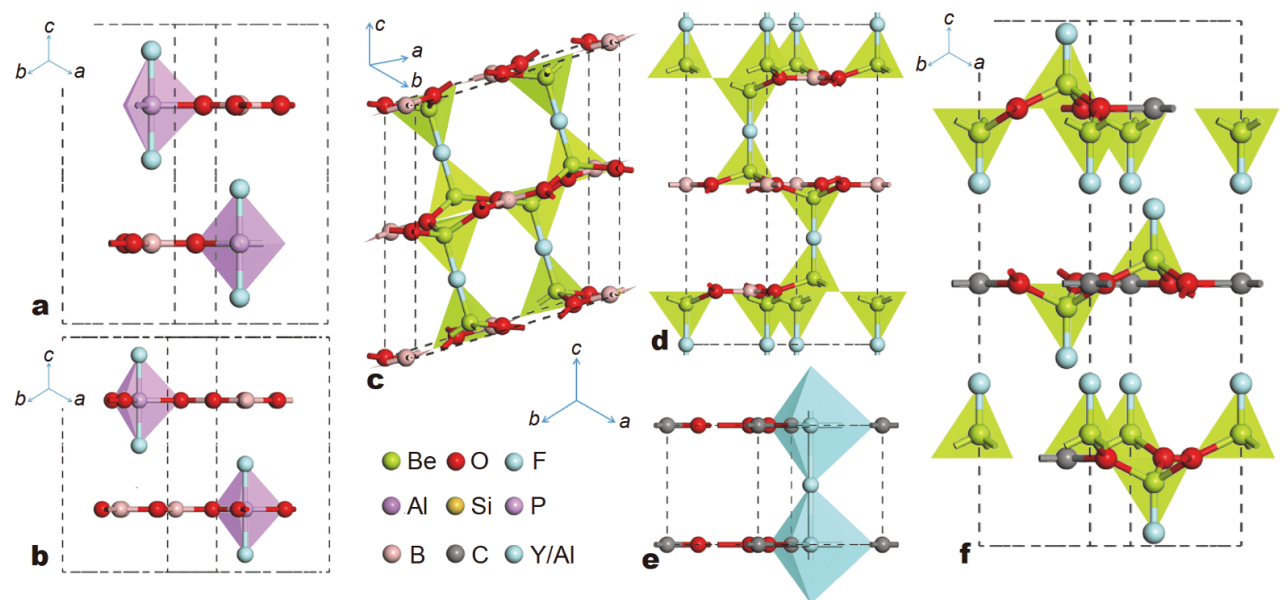

Figure 5 Structures of $\mathrm{PBO}_{3} \mathrm{~F}_{2}(\mathrm{a}), \mathrm{PB}_{3} \mathrm{O}_{6} \mathrm{~F}_{2}(\mathrm{~b}), \boldsymbol{\alpha}-\mathrm{Be}_{2} \mathrm{BO}_{3} \mathrm{~F}(\mathrm{c}), \gamma-\mathrm{Be}_{2} \mathrm{BO}_{3} \mathrm{~F}(\mathrm{~d}), \mathrm{Y} / \mathrm{AlCO}_{3} \mathrm{~F}$ (e) and $\mathrm{Be}_{2} \mathrm{CO}_{3} \mathrm{~F}_{2}$ (f).

$\lambda_{\mathrm{PM}}(\sim 180 \mathrm{~nm})$, but small SHG effect $(\sim 0.2 \times \mathrm{KBBF})[82]$. The adjacent $\left(\mathrm{Be}_{2} \mathrm{BO}_{3} \mathrm{~F}_{2}\right)_{\infty}$ layers are interconnected by the bridge $\mathrm{F}^{-}$anions, resulting in densely stacked layers. If the borate groups are parallel-aligned as illustrated in Fig. 5d, the total SHG response will be strengthened. From our calculations in Table 1 , the $\Delta n$ and $d_{12}$ of the designed $\gamma$-phase are 0.10 and $1.5 \times \mathrm{KBBF}$, respectively [36]. Its $\lambda_{\mathrm{PM}}$ can reach $149 \mathrm{~nm}$, blue-shifted by about $12 \mathrm{~nm}$ than that of KBBF. Fortunately, this sample was obtained in the experiments and reported recently [37]. The available experimental measurements are consistent with our predictions, guaranteeing our modeling system suitable to some extent.

Some similar F-bridge layer-framework systems can be also designed theoretically, e.g., $\mathrm{YCO}_{3} \mathrm{~F}$ and $\mathrm{AlCO}_{3} \mathrm{~F}$ as shown in Fig. 5e. Based on the existing NLO carbonate $\mathrm{YCO}_{3} \mathrm{OH}$ with $E_{\mathrm{g}} \approx 7.2 \mathrm{eV}, d_{36} \approx \mathrm{KDP}, \Delta n \approx 0.06$ and $\lambda_{\mathrm{PM}}$ $\approx 255 \mathrm{~nm}$, we have constructed a layer $\mathrm{YCO}_{3} \mathrm{~F}$ structure by introducing $\mathrm{F}$ instead of $\mathrm{OH}$ to enlarge the polarity and anisotropy [83]. From the DFT calculations as listed in Table 1, indeed the NLO response and $\Delta n$ are greatly enlarged, respectively from 0.5 to $2.6 \mathrm{pm} \mathrm{V}^{-1}$ and 0.06 to $0.20 \mathrm{pm} \mathrm{V}^{-1}$. Accordingly, $\mathrm{YCO}_{3} \mathrm{~F}$ can realize the practical DUV $177.3 \mathrm{~nm}$ SHG output since its $\lambda_{\mathrm{PM}} \approx 168 \mathrm{~nm}$. In order to further enlarge $E_{\mathrm{g}}$, we can introduce $\mathrm{Al}^{3+}$ to replace $\mathrm{Y}^{3+}$ to coordinate with $\left(\mathrm{CO}_{3}\right)^{2-}$. The results show that $E_{\mathrm{g}}$ of $\mathrm{AlCO}_{3} \mathrm{~F}$ is actually increased while maintaining $\Delta n$ sufficiently large ( 0.15). Thus, $\lambda_{\mathrm{PM}}$ of $\mathrm{AlCO}_{3} \mathrm{~F}$ is blueshifted from 168 to $146 \mathrm{~nm}$. Meanwhile, the NLO coefficient is $2.5 \times \mathrm{KDP}$, available for the sufficient DUV conversion. All the analysis indicates that the polar Fbridge layer-frameworks without $A$-site cations are indeed beneficial to the DUV PM output with sufficient
NLO effect.

$v d W$-connected layer-structures and $\mathrm{Be}_{2} \mathrm{CO}_{3} \mathrm{~F}_{2}$

The dense-stacking layer-frameworks without $A$-site cations belong to another kind of DUV NLO structures. The key strategy is to use the interlayer van der Waals $(\mathrm{vdW})$ interaction instead of conversional ionic interaction to make the adjacent layers connected densely. The kind of vdW layers is demonstrated in Fig. 5f, in which we introduce the $\left(\mathrm{Be}_{2} \mathrm{CO}_{3} \mathrm{~F}_{2}\right)_{\infty}$ carbonate layers to replace the $\left(\mathrm{Be}_{2} \mathrm{BO}_{3} \mathrm{~F}_{2}\right)_{\infty}$ borate layers in KBBF [77]. Note that $\left(\mathrm{Be}_{2} \mathrm{CO}_{3} \mathrm{~F}_{2}\right)_{\infty}$ itself is an NLO-active layer structure with large anisotropy, polar alignment and few nonbonding states. As the produced crystal, $\mathrm{Be}_{2} \mathrm{CO}_{3} \mathrm{~F}_{2}$ can exhibit large $\Delta n \approx 0.11$, strong $d_{11} \approx 2 \times \mathrm{KBBF}$, and short $\lambda_{\mathrm{PM}} \approx$ $155 \mathrm{~nm}$ (Table 1), which is expected to be a possible DUV NLO system. Moreover, its type-I (o+oàe) PM angle $\theta$ at $177.3 \mathrm{~nm}$ is about $56^{\circ}$, so its $d_{\text {eff }}$ is about 3.3 times larger than that of KBBF. Under the same laser and crystal conditions, its output power would be about 10 times larger than that of KBBF.

There are no interlayer atoms in $\mathrm{Be}_{2} \mathrm{CO}_{3} \mathrm{~F}_{2}$, resulting in the more densely stacked layers. We can also design similar vdW layers such as $\mathrm{SiCO}_{3} \mathrm{~F}_{2}$, which consists of polar $\left(\mathrm{SiCO}_{3} \mathrm{~F}_{2}\right)_{\infty}$ layers formed by $\left(\mathrm{SiO}_{3} \mathrm{~F}_{2}\right)^{4-}$ and $\left(\mathrm{CO}_{3}\right)^{2-}$. Its NLO properties are listed in Table 1, including short $\lambda_{\mathrm{UV}}$ $(\sim 129 \mathrm{~nm})$, strong $d_{i j}\left(\sim 1 \mathrm{pm} \mathrm{V}^{-1}\right)$ and large $\Delta n(\sim 0.13)$. Compared with $\mathrm{Be}_{2} \mathrm{CO}_{3} \mathrm{~F}_{2}, \mathrm{SiCO}_{3} \mathrm{~F}_{2}$ exhibits a larger $E_{\mathrm{g}}$ due to the stronger covalence of $\mathrm{Si}$ than Be. Considering the close $d_{i j}$ and $\Delta n$, it can realize a shorter DUV output $\left(\lambda_{\mathrm{PM}} \approx 129 \mathrm{~nm}\right)$, which is the theoretically shortest wavelength in the DUV NLO crystal field. Other structures include $\mathrm{AlNO}_{3} \mathrm{~F}_{2}, \mathrm{PBO}_{3} \mathrm{~F}_{2}$ and $\mathrm{PB}_{3} \mathrm{O}_{6} \mathrm{~F}_{2}$ as listed in 
Table 1, indicating the possible DUV NLO properties induced by polar vdW layer structures without $A$-site cations [77].

\section{CONCLUSIONS}

In summary, we systematically evaluated the DUV NLO capabilities induced by the $A$-site, $B$-site, $G$-site and $H$ site parts in the KBBF-type structures. These studies are mainly based on our developed computer-aided modeling system, which are efficiently accurate and can serve as a blueprint for DUV NLO materials exploration and design. Importantly, high-throughput calculations can also be performed in other broader systems according to our proposed modeling and evaluation flowchart. In the current stage, finding a compound with possible DUV NLO properties from ICSD is of great difficulty. Therefore, the comprehensive materials design and analysis using effective theoretical modeling proposal is urgently necessary for current and future DUV NLO researches $[84,85]$.

As summarized in Fig. 6, dozens of typical KBBF-type structures, regardless existing or hypothetical, are evaluated based on the structure-selections and first-principles calculations (for details see Table S1). The evaluation standard of DUV NLO crystals is finalized and further illustrated by the coordinate of the shortest SHG wavelength $\lambda_{\mathrm{PM}}$ and SHG effect $d_{i j}$. As the practical laser sources, 193.7 and $177.3 \mathrm{~nm}$ are selected as two important redlines for DUV generation. Moreover, KBBF is marked by black stars as the benchmark DUV NLO crystal $\left(d_{22}=0.45 \mathrm{pm} \mathrm{V}^{-1} v s\right.$. $\left.\lambda_{\mathrm{PM}}=161 \mathrm{~nm}\right)$. $\mathrm{BBO}$ and $\mathrm{YAl}_{3}\left(\mathrm{BO}_{3}\right)_{4}(\mathrm{YAB})$, which are two typical NLO materials in the DUV region, are used as two referenced NLO crystals with $2 \times \mathrm{KBBF} v s .204 \mathrm{~nm}$ and $3.9 \times \mathrm{KBBF} v$ s. $210 \mathrm{~nm}$, respectively.

Circular dots are those experimentally obtained crystal structures. Among them, 14 compounds including RBBF [57], CBBF [58], NBBF [59], $\mathrm{ABBF}$ [37], $\mathrm{BaBe}_{2} \mathrm{BO}_{3} \mathrm{~F}_{3}$ [65], $\mathrm{MB}_{5} \mathrm{O}_{7} \mathrm{~F}_{3}(\mathrm{M}=\mathrm{Ca}, \mathrm{Sr})$ [43], $\mathrm{NaB}_{4} \mathrm{O}_{6} \mathrm{~F}$ [86], $\mathrm{RbB}_{4} \mathrm{O}_{6} \mathrm{~F}$ [41], $\mathrm{CsB}_{4} \mathrm{O}_{6} \mathrm{~F}$ [39], $\mathrm{CsKB}_{8} \mathrm{O}_{12} \mathrm{~F}_{2}$ [41], $\alpha$-BBF [82], $\gamma$-BBF [37] and $\mathrm{ABF}$ [40] exhibit possible DUV NLO properties from available experimental and calculated results. In particular, $\gamma$-BBF and ABF are expected to have superior DUV SHG capabilities than KBBF. Therein, both of them are located at the preferred area of DUV NLO materials with shorter $\lambda_{\mathrm{PM}}(<161 \mathrm{~nm})$ and larger $\left|d_{i j}\right|_{\max }(>\mathrm{KBBF})$ in the green shadow of Fig. 6 . Red pentagrams are our designed systems, including 12 compounds proposed in the previous studies and 18 new structures predicted in this work. Among them, 20 crystals exhibit possible DUV NLO output properties with six located in the preferred

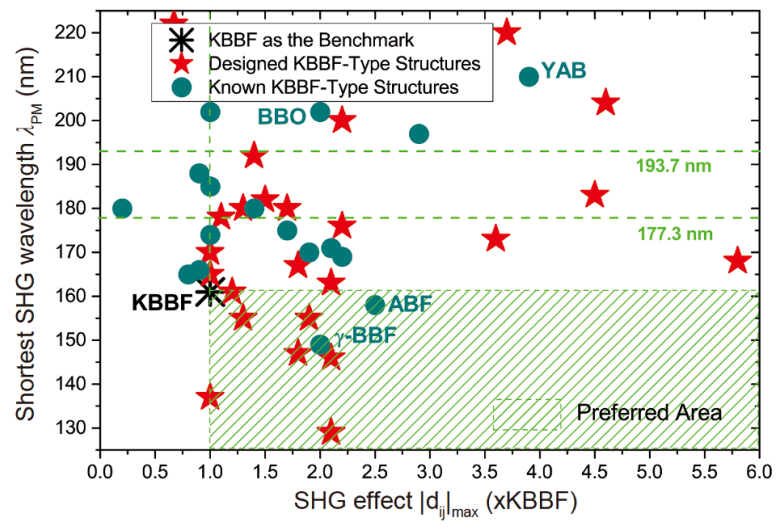

Figure 6 Evaluation standard of DUV NLO crystals by the coordination of $d_{i j}$ and $\lambda_{\mathrm{PM}}$. DUV NLO properties of the typical existing and designed structures are evaluated. The preferred area is shown as the green shadow.

area. In particular, $\mathrm{ABBF}$ and $\gamma-\mathrm{BBF}$ are two successful systems that were first predicted theoretically and then obtained experimentally. It should be pointed out that quasi-phase matching technique is also a very promising method for the DUV generation, especially for those NLO crystals with small birefringence but large optical nonlinearity (e.g., $\mathrm{SrB}_{4} \mathrm{O}_{7}$ or $\mathrm{BPO}_{4}$ ) [72,79]. Although it may have higher requirements for the engineering and design of NLO materials, once realized, it will promote the development of DUV laser field [87].

Depending on the computer-aided modeling system, we predict seven novel structures for the first time including $\quad \mathrm{KAlBO}_{3} \mathrm{~F}, \quad \mathrm{KAl}_{2} \mathrm{~B}_{2} \mathrm{O}_{6} \mathrm{~F}, \quad \mathrm{KScBO}_{3} \mathrm{~F}, \quad \mathrm{~KB}_{2} \mathrm{O}_{3} \mathrm{~F}$, $\mathrm{KB}_{4} \mathrm{O}_{6} \mathrm{~F}, \mathrm{KSiBO}_{3} \mathrm{~F}_{2}$ and $\mathrm{KSiB}_{3} \mathrm{O}_{6} \mathrm{~F}_{2}$ that can exhibit good DUV NLO properties as shown in Table 1. If we further perform cationic replacement using $\mathrm{Rb}, \mathrm{Cs}, \mathrm{NH}_{4}$ instead of $\mathrm{K}$, more NLO compounds with similar properties could be found according to the NLO size-effect. Together with some other systems that we studied previously, e.g., $\mathrm{RbAlCO}_{3} \mathrm{~F}_{2}, \mathrm{BaB}_{4} \mathrm{O}_{6} \mathrm{~F}_{2}, \mathrm{AlNO}_{3} \mathrm{~F}_{2}, \mathrm{PBO}_{3} \mathrm{~F}_{2}$, $\mathrm{PB}_{3} \mathrm{O}_{6} \mathrm{~F}_{2}, \mathrm{YCO}_{3} \mathrm{~F}, \mathrm{Be}_{2} \mathrm{CO}_{3} \mathrm{~F}_{2}$ and $\mathrm{SiCO}_{3} \mathrm{~F}_{2}[35,74,77,81]$, we have acquired several series of possible DUV NLO crystals by the design blueprint, looking forward to further exploration in the experiments. Based on the findings of more DUV NLO structural types, we believe that our proposed first-principles-based modeling system and design blueprint will play a more positive role in the exploration for new DUV NLO materials.

\section{Received 28 March 2020; accepted 22 April 2020;} published online 4 June 2020

1 Kiss T, Kanetaka F, Yokoya T, et al. Photoemission spectroscopic evidence of gap anisotropy in an f-electron superconductor. Phys 
Rev Lett, 2005, 94: 057001

2 Savage N. Ultraviolet lasers. Nat Photon, 2007, 2007: 83-85

3 Kanai T, Wang X, Adachi S, et al. Watt-level tunable deep ultraviolet light source by a KBBF prism-coupled device. Opt Express, 2009, 17: 8696-8703

4 Petrov V. Frequency down-conversion of solid-state laser sources to the mid-infrared spectral range using non-oxide nonlinear crystals. Prog Quantum Electron, 2015, 42: 1-106

5 Boyd RW, Nonlinear Optics. 3rd Edition. San Diego: Academic Press, 2008

6 Li M, Pan H, Tong Y, et al. All-optical ultrafast polarization switching of terahertz radiation by impulsive molecular alignment. Opt Lett, 2011, 36: 3633-3635

7 Yin $\mathrm{X}$, Ye Z, Chenet DA, et al. Edge nonlinear optics on a $\mathrm{MoS}_{2}$ atomic monolayer. Science, 2014, 344: 488-490

8 Alam MZ, De Leon I, Boyd RW. Large optical nonlinearity of indium tin oxide in its epsilon-near-zero region. Science, 2016, 352: 795-797

9 Wu L, Patankar S, Morimoto T, et al. Giant anisotropic nonlinear optical response in transition metal monopnictide Weyl semimetals. Nat Phys, 2017, 13: 350-355

10 Gallagher SM, Albrecht AW, Hybl JD, et al. Heterodyne detection of the complete electric field of femtosecond four-wave mixing signals. J Opt Soc Am B, 1998, 15: 2338

11 Sekikawa T, Kosuge A, Kanai T, et al. Nonlinear optics in the extreme ultraviolet. Nature, 2004, 432: 605-608

12 Cyranoski D. Materials science: China's crystal cache. Nature, 2009, 457: 953-955

13 Eismann U, Scholz M, Paasch-Colberg T, et al. Short, shorter, shortest: Diode lasers in the deep ultraviolet. Laser Focus World, 2016, 52: 39-44

14 Kiss T, Shimojima T, Kanetaka F, et al. Ultrahigh-resolution photoemission spectroscopy of superconductors using a VUV laser. J Electron Spectr Related Phenomena, 2005, 144: 953-956

15 Koralek JD, Douglas JF, Plumb NC, et al. Laser based angle-resolved photoemission, the sudden approximation, and quasiparticle-like spectral peaks in $\mathrm{Bi}_{2} \mathrm{Sr}_{2} \mathrm{CaCu}_{2} \mathrm{O}_{8+\delta}$. Phys Rev Lett, 2006, 96: 017005

16 Meng J, Liu G, Zhang W, et al. Coexistence of Fermi arcs and Fermi pockets in a high- $T_{\mathrm{c}}$ copper oxide superconductor. Nature, 2009, 462: 335-338

17 Chen TA, Chuu CP, Tseng CC, et al. Wafer-scale single-crystal hexagonal boron nitride monolayers on $\mathrm{Cu}$ (111). Nature, 2020, 579: 219-223

18 Chen C, Sasaki T, Li R, et al. Nonlinear Optical Borate Crystals: Principles and Applications. Weinheim: Wiley-VCH, 2012

19 Zhang W, Yu H, Wu H, et al. Phase-matching in nonlinear optical compounds: a materials perspective. Chem Mater, 2017, 29: 26552668

20 Halasyamani PS, Rondinelli JM. The must-have and nice-to-have experimental and computational requirements for functional frequency doubling deep-UV crystals. Nat Commun, 2018, 9: 2972

21 Chen C, Lin Z, Wang Z. The development of new borate-based UV nonlinear optical crystals. Appl Phys B, 2005, 80: 1-25

22 Yao W, He R, Wang X, et al. Analysis of deep-UV nonlinear optical borates: approaching the end. Adv Opt Mater, 2014, 2: 411417

23 Tran TT, Yu H, Rondinelli JM, et al. Deep ultraviolet nonlinear optical materials. Chem Mater, 2016, 28: 5238-5258

24 Chen $\mathrm{C}, \mathrm{Wu} \mathrm{Y}$, Jiang $\mathrm{A}$, et al. New nonlinear-optical crystal:
$\mathrm{LiB}_{3} \mathrm{O}_{5}$. J Opt Soc Am B, 1989, 6: 616-621

25 Chen $\mathrm{CT}$, Wu BC, Jiang AD, et al. A new-type ultraviolet SHG crystal-beta- $\mathrm{BaB}_{2} \mathrm{O}_{4}$. Sci Sin Ser B, 1985, 28: 235-243

26 Chen CT, Wang GL, Wang XY, et al. Deep-UV nonlinear optical crystal $\mathrm{KBe}_{2} \mathrm{BO}_{3} \mathrm{~F}_{2}$-discovery, growth, optical properties and applications. Appl Phys B, 2009, 97: 9-25

27 Xu B, Liu L, Wang X, et al. Generation of high power $200 \mathrm{~mW}$ laser radiation at $177.3 \mathrm{~nm}$ in $\mathrm{KBe}_{2} \mathrm{BO}_{3} \mathrm{~F}_{2}$ crystal. Appl Phys B, 2015, 121: 489-494

28 Kang L, Liang F, Jiang X, et al. First-principles design and simulations promote the development of nonlinear optical crystals. Acc Chem Res, 2019, 53: 209-217

29 Chen C, Ye N, Lin J, et al. Computer-assisted search for nonlinear optical crystals. Adv Mater, 1999, 11: 1071-1078

30 Lin J, Lee MH, Liu ZP, et al. Mechanism for linear and nonlinear optical effects in $\beta-\mathrm{BaB}_{2} \mathrm{O}_{4}$ crystals. Phys Rev B, 1999, 60: 1338013389

31 He R, Lin ZS, Lee MH, et al. Ab initio studies on the mechanism for linear and nonlinear optical effects in $\mathrm{YAl}_{3}\left(\mathrm{BO}_{3}\right)_{4}$. J Appl Phys, 2011, 109: 103510

32 Lin ZS, Kang L, Zheng T, et al. Strategy for the optical property studies in ultraviolet nonlinear optical crystals from density functional theory. Comput Mater Sci, 2012, 60: 99-104

33 He R, Huang H, Kang L, et al. Bandgaps in the deep ultraviolet borate crystals: prediction and improvement. Appl Phys Lett, 2013, 102: 231904

34 Lin Z, Jiang X, Kang L, et al. First-principles materials applications and design of nonlinear optical crystals. J Phys D-Appl Phys, 2014, 47: 253001

35 Kang L, Luo S, Huang H, et al. Prospects for fluoride carbonate nonlinear optical crystals in the UV and Deep-UV regions. J Phys Chem C, 2013, 117: 25684-25692

36 Kang L, Luo S, Peng G, et al. First-principles design of a deepultraviolet nonlinear-optical crystal from $\mathrm{KBe}_{2} \mathrm{BO}_{3} \mathrm{~F}_{2}$ to $\mathrm{NH}_{4} \mathrm{Be}_{2} \mathrm{BO}_{3} \mathrm{~F}_{2}$. Inorg Chem, 2015, 54: 10533-10535

37 Peng G, Ye N, Lin Z, et al. $\mathrm{NH}_{4} \mathrm{Be}_{2} \mathrm{BO}_{3} \mathrm{~F}_{2}$ and $\gamma-\mathrm{Be}_{2} \mathrm{BO}_{3} \mathrm{~F}$ : Overcoming the layering habit in $\mathrm{KBe}_{2} \mathrm{BO}_{3} \mathrm{~F}_{2}$ for the next-generation deep-ultraviolet nonlinear optical materials. Angew Chem Int Ed, 2018, 57: 8968-8972

38 Zhang B, Shi G, Yang Z, et al. Fluorooxoborates: beryllium-free deep-ultraviolet nonlinear optical materials without layered growth. Angew Chem Int Ed, 2017, 56: 3916-3919

39 Wang $\mathrm{X}$, Wang $\mathrm{Y}$, Zhang $\mathrm{B}$, et al. $\mathrm{CsB}_{4} \mathrm{O}_{6} \mathrm{~F}$ : A congruent-melting deep-ultraviolet nonlinear optical material by combining superior functional units. Angew Chem Int Ed, 2017, 56: 14119-14123

40 Shi G, Wang Y, Zhang F, et al. Finding the next deep-ultraviolet nonlinear optical material: $\mathrm{NH}_{4} \mathrm{~B}_{4} \mathrm{O}_{6} \mathrm{~F}$. J Am Chem Soc, 2017, 139: 10645-10648

41 Wang Y, Zhang B, Yang Z, et al. Cation-tuned synthesis of fluorooxoborates: towards optimal deep-ultraviolet nonlinear optical materials. Angew Chem Int Ed, 2018, 57: 2150-2154

42 Mutailipu $\mathrm{M}$, Zhang $\mathrm{M}$, Zhang $\mathrm{B}$, et al. $\mathrm{SrB}_{5} \mathrm{O}_{7} \mathrm{~F}_{3}$ functionalized with $\left[\mathrm{B}_{5} \mathrm{O}_{9} \mathrm{~F}_{3}\right]^{6-}$ chromophores: Accelerating the rational design of deep-ultraviolet nonlinear optical materials. Angew Chem Int Ed, 2018, 57: 6095-6099

43 Luo $\mathrm{M}$, Liang $\mathrm{F}$, Song $\mathrm{Y}$, et al. $\mathrm{M}_{2} \mathrm{~B}_{10} \mathrm{O}_{14} \mathrm{~F}_{6}(\mathrm{M}=\mathrm{Ca}$, Sr): Two noncentrosymmetric alkaline earth fluorooxoborates as promising next-generation deep-ultraviolet nonlinear optical materials. J Am Chem Soc, 2018, 140: 3884-3887

44 Andriyevsky B, Doll K, Cakmak G, et al. DFT-based ab initio study 
of structural and electronic properties of lithium fluorooxoborate $\mathrm{LiB}_{6} \mathrm{O}_{9} \mathrm{~F}$ and experimentally observed second harmonic generation. Phys Rev B, 2011, 84: 125112

45 Jain A, Ong SP, Hautier G, et al. Commentary: The Materials Project: A materials genome approach to accelerating materials innovation. APL Mater, 2013, 1: 011002

Jiang X, Luo S, Kang L, et al. First-principles evaluation of the alkali and/or alkaline earth beryllium borates in deep ultraviolet nonlinear optical applications. ACS Photonics, 2015, 2: 1183-1191

47 Xia Y, Chen C, Tang D, et al. New nonlinear optical crystals for UV and VUV harmonic generation. Adv Mater, 1995, 7: 79-81

48 Clark SJ, Segall MD, Pickard CJ, et al. First principles methods using CASTEP. Z für Kristallographie - Crystline Mater, 2005, 5-6: 567-570

49 Kohn W, Sham LJ. Self-consistent equations including exchange and correlation effects. Phys Rev, 1965, 140: A1133-A1138

50 Rappe AM, Rabe KM, Kaxiras E, et al. Optimized pseudopotentials. Phys Rev B, 1990, 41: 1227-1230

51 Pfrommer BG, Côté M, Louie SG, et al. Relaxation of crystals with the quasi-Newton method. J Comput Phys, 1997, 131: 233-240

52 Adamo C, Barone V. Toward reliable density functional methods without adjustable parameters: the PBE0 model. J Chem Phys, 1999, 110: 6158-6170

53 Perdew JP, Burke K, Ernzerhof M. Generalized gradient approximation made simple. Phys Rev Lett, 1996, 77: 3865-3868

54 Baroni S, de Gironcoli S, Dal Corso A, et al. Phonons and related crystal properties from density-functional perturbation theory. Rev Mod Phys, 2001, 73: 515-562

55 Payne MC, Teter MP, Allan DC, et al. Iterative minimization techniques for $a b$ initio total-energy calculations: molecular dynamics and conjugate gradients. Rev Mod Phys, 1992, 64: 10451097

56 Kang L, Luo S, Huang $\mathrm{H}$, et al. Ab initio studies on the optical effects in the deep ultraviolet nonlinear optical crystals of the $\mathrm{KBe}_{2} \mathrm{BO}_{3} \mathrm{~F}_{2}$ family. J Phys-Condens Matter, 2012, 24: 335503

57 Chen $\mathrm{C}$, Luo $\mathrm{S}$, Wang $\mathrm{X}$, et al. Deep UV nonlinear optical crystal: $\mathrm{RbBe}_{2}\left(\mathrm{BO}_{3}\right) \mathrm{F}_{2}$. J Opt Soc Am B, 2009, 26: 1519-1525

58 Huang $\mathrm{H}$, Chen $\mathrm{C}$, Wang $\mathrm{X}$, et al. Ultraviolet nonlinear optical crystal: $\mathrm{CsBe}_{2} \mathrm{BO}_{3} \mathrm{~F}_{2}$. J Opt Soc Am B, 2011, 28: 2186-2190

59 Guo S, Kang L, Liu L, et al. Deep-ultraviolet nonlinear optical crystal $\mathrm{NaBe}_{2} \mathrm{BO}_{3} \mathrm{~F}_{2}-$ Structure, growth and optical properties. J Cryst Growth, 2019, 518: 45-50

60 McMillen $\mathrm{CD}, \mathrm{Hu} \mathrm{J}$, VanDerveer $\mathrm{D}$, et al. Trigonal structures of $\mathrm{ABe}_{2} \mathrm{BO}_{3} \mathrm{~F}_{2}(A=\mathrm{Rb}, \mathrm{Cs}, \mathrm{Tl})$ crystals. Acta Crystlogr B Struct Sci, 2009, 65: 445-449

61 Chen C, Wang Y, Wu B, et al. Design and synthesis of an ultraviolet-transparent nonlinear optical crystal $\mathrm{Sr}_{2} \mathrm{Be}_{2} \mathrm{~B}_{2} \mathrm{O}_{7}$. Nature, 1995, 373: 322-324

62 Huang H, Yao J, Lin Z, et al. Molecular engineering design to resolve the layering habit and polymorphism problems in deep UV NLO crystals: New structures in $\mathrm{MM}^{\prime} \mathrm{Be}_{2} \mathrm{~B}_{2} \mathrm{O}_{6} \mathrm{~F}\left(\mathrm{M}=\mathrm{Na}, \mathrm{M}^{\prime}=\mathrm{Ca}\right.$; $\mathrm{M}=\mathrm{K}, \mathrm{M}^{\prime}=\mathrm{Ca}$, Sr). Chem Mater, 2011, 23: 5457-5463

63 Huang $\mathrm{H}$, Yao J, Lin Z, et al. $\mathrm{NaSr}_{3} \mathrm{Be}_{3} \mathrm{~B}_{3} \mathrm{O}_{9} \mathrm{~F}_{4}$ : A promising deepultraviolet nonlinear optical material resulting from the cooperative alignment of the $\left[\mathrm{Be}_{3} \mathrm{~B}_{3} \mathrm{O}_{12} \mathrm{~F}\right]^{10-}$ anionic group. Angew Chem Int Ed, 2011, 50: 9141-9144

64 Wang X, Liu L, Wang X, et al. Growth and optical properties of the novel nonlinear optical crystal $\mathrm{NaSr}_{3} \mathrm{Be}_{3} \mathrm{~B}_{3} \mathrm{O}_{9} \mathrm{~F}_{4}$. CrystEngComm, 2015, 17: 925-929

65 Guo S, Jiang X, Liu L, et al. $\mathrm{BaBe}_{2} \mathrm{BO}_{3} \mathrm{~F}_{3}$ : A KBBF-type deep- ultraviolet nonlinear optical material with reinforced $\left[\mathrm{Be}_{2} \mathrm{BO}_{3} \mathrm{~F}_{2}\right]_{\infty}$ layers and short phase-matching wavelength. Chem Mater, 2016, 28: 8871-8875

$66 \mathrm{Hu} \mathrm{Z}$, Yue Y, Chen X, et al. Growth and structure redetermination of a nonlinear $\mathrm{BaAlBO}_{3} \mathrm{~F}_{2}$ crystal. Solid State Sci, 2011, 13: 875-878

67 Zhao S, Gong P, Luo S, et al. Beryllium-free $\mathrm{Rb}_{3} \mathrm{Al}_{3} \mathrm{~B}_{3} \mathrm{O}_{10} \mathrm{~F}$ with reinforced interlayer bonding as a deep-ultraviolet nonlinear optical crystal. J Am Chem Soc, 2015, 137: 2207-2210

68 Zhao S, Kang L, Shen Y, et al. Designing a beryllium-free deepultraviolet nonlinear optical material without a structural instability problem. J Am Chem Soc, 2016, 138: 2961-2964

$69 \mathrm{Li} \mathrm{RK}$, Chen P. Cation coordination control of anionic group alignment to maximize SHG effects in the $\mathrm{BaMBO}_{3} \mathrm{~F}(\mathrm{M}=\mathrm{Zn}, \mathrm{Mg})$ series. Inorg Chem, 2010, 49: 1561-1565

70 Yan $\mathrm{X}$, Luo $\mathrm{S}$, Lin $\mathrm{Z}$, et al. $\mathrm{LaBeB}_{3} \mathrm{O}_{7}$ : a new phase-matchable nonlinear optical crystal exclusively containing the tetrahedral $\mathrm{XO}_{4}$ ( $\mathrm{X}=\mathrm{B}$ and Be) anionic groups. J Mater Chem C, 2013, 1: 36163622

71 Yan $\mathrm{X}$, Luo $\mathrm{S}$, Lin Z, et al. $\mathrm{ReBe}_{2} \mathrm{~B}_{5} \mathrm{O}_{11}(\mathrm{Re}=\mathrm{Y}, \mathrm{Gd})$ : Rare-earth beryllium borates as deep-ultraviolet nonlinear-optical materials. Inorg Chem, 2014, 53: 1952-1954

72 Pan F, Shen G, Wang R, et al. Growth, characterization and nonlinear optical properties of $\mathrm{SrB}_{4} \mathrm{O}_{7}$ crystals. J Cryst Growth, 2002, 241: 108-114

73 Becker P. Borate materials in nonlinear optics. Adv Mater, 1998, 10: 979-992

74 Liang F, Kang L, Gong P, et al. Rational design of deep-ultraviolet nonlinear optical materials in fluorooxoborates: toward optimal planar configuration. Chem Mater, 2017, 29: 7098-7102

75 Zou G, Ye N, Huang L, et al. Alkaline-alkaline earth fluoride carbonate crystals $\mathrm{ABCO}_{3} \mathrm{~F}(\mathrm{~A}=\mathrm{K}, \mathrm{Rb}, \mathrm{Cs} ; \mathrm{B}=\mathrm{Ca}, \mathrm{Sr}, \mathrm{Ba})$ as nonlinear optical materials. J Am Chem Soc, 2011, 133: 2000120007

76 Tran TT, He J, Rondinelli JM, et al. $\mathrm{RbMgCO}_{3} \mathrm{~F}$ : A new berylliumfree deep-ultraviolet nonlinear optical material. J Am Chem Soc, 2015, 137: 10504-10507

77 Kang L, Lin Z, Liu F, et al. Removal of A-site alkali and alkaline earth metal cations in $\mathrm{KBe}_{2} \mathrm{BO}_{3} \mathrm{~F}_{2}$-type layered structures to enhance the deep-ultraviolet nonlinear optical capability. Inorg Chem, 2018, 57: 11146-11156

78 Liang F, Kang L, Zhang X, et al. Molecular construction using $\left(\mathrm{C}_{3} \mathrm{~N}_{3} \mathrm{O}_{3}\right)^{3-}$ anions: Analysis and prospect for inorganic metal cyanurates nonlinear optical materials. Cryst Growth Des, 2017, 17: 4015-4020

79 Li Z, Lin Z, Wu Y, et al. Crystal growth, optical properties measurement, and theoretical calculation of $\mathrm{BPO}_{4}$. Chem Mater, 2004, 16: 2906-2908

80 Parise JB, Gier TE. Hydrothermal syntheses and structural refinements of single crystal lithium boron germanate and silicate, $\mathrm{LiBGeO}_{4}$ and $\mathrm{LiBSiO}_{4}$. Chem Mater, 1992, 4: 1065-1067

81 Kang L, Liang F, Gong P, et al. Two novel deep-ultraviolet nonlinear optical crystals with shorter phase-matching second harmonic generation than $\mathrm{KBe}_{2} \mathrm{BO}_{3} \mathrm{~F}_{2}$ : A first-principles prediction. Phys Status Solidi RRL, 2018, 18: 1800276

82 Zhang X, Guan RF, Wu DQ, et al. Enzyme immobilization on amino-functionalized mesostructured cellular foam surfaces, characterization and catalytic properties. J Mol Catal B-Enzymatic, 2005, 33: 43-50

83 Beall GW, Milligan WO, Mroczkowski S. Yttrium carbonate hydroxide. Acta Crystlogr B Struct Crystlogr Cryst Chem, 1976, 32: 
3143-3144

84 Yang Z, Tudi A, Lei BH, et al. Enhanced nonlinear optical functionality in birefringence and refractive index dispersion of the deep-ultraviolet fluorooxoborates. Sci China Mater, 2020, 63: 1480-1488

85 Wei Z, Zhang W, Zeng $\mathrm{H}$, et al. Prediction of ternary fluorooxoborates with coplanar triangular units $\left[\mathrm{BO}_{x} \mathrm{~F}_{3-x}\right]^{x-}$ from firstprinciples. Dalton Trans, 2020, 49: 5424-5428

86 Zhang Z, Wang Y, Zhang B, et al. Polar fluorooxoborate, $\mathrm{NaB}_{4} \mathrm{O}_{6} \mathrm{~F}$ : A promising material for ionic conduction and nonlinear optics. Angew Chem Int Ed, 2018, 57: 6577-6581

87 Trabs P, Noack F, Aleksandrovsky AS, et al. Generation of coherent radiation in the vacuum ultraviolet using randomly quasiphase-matched strontium tetraborate. Opt Lett, 2016, 41: 618-621

Acknowledgements This work was supported by the National Natural Science Foundation of China (NSFC 51872297, 51890864, 11574024), and NSAF (U1930402). Lin Z acknowledges support from outstanding member in Youth Innovation Promotion Association at the Chinese Academy of Sciences (CAS), and Fujian Institute of Innovation (FJCXY18010201) in CAS

Author contributions Kang L performed the theoretical calculations. Kang L, Lin Z and Huang B supervised the theoretical analysis and wrote the paper. Liang $\mathrm{F}$ gave the academic suggestion. All authors contributed to the general discussion.

Conflict of interest The authors declare that they have no conflict of interest.

Supplementary information Phonon vibration spectra of $\mathrm{NaBe}_{2} \mathrm{BO}_{3} \mathrm{~F}_{2}$, $\mathrm{LiBe}_{2} \mathrm{BO}_{3} \mathrm{~F}_{2}, \mathrm{KAlBO}_{3} \mathrm{~F}, \mathrm{KAl}_{2} \mathrm{~B}_{2} \mathrm{O}_{6} \mathrm{~F}, \mathrm{KBe}_{2} \mathrm{~B}_{3} \mathrm{O}_{6} \mathrm{~F}_{2}, \mathrm{BaAlB}_{3} \mathrm{O}_{6} \mathrm{~F}_{2}, \mathrm{AlCO}_{3} \mathrm{~F}$, $\mathrm{SiCO}_{3} \mathrm{~F}_{2}, \mathrm{AlNO}_{3} \mathrm{~F}_{2}, \mathrm{PBO}_{3} \mathrm{~F}_{2}$, and $\mathrm{PB}_{3} \mathrm{O}_{6} \mathrm{~F}_{2}$, and NLO properties including $d_{i j}$ and $\lambda_{\mathrm{PM}}$ of the compounds in Fig. 6 are available in the online version of the paper.

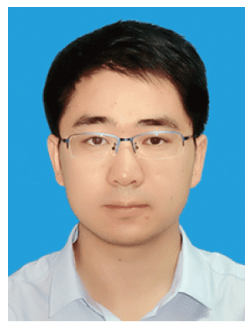

Lei Kang received his $\mathrm{BSc}$ in physics from Shandong University, and $\mathrm{PhD}$ in condensed matter physics from the Technical Institute of Physics and Chemistry (TIPC), Chinese Academy of Sciences (CAS) in 2016. He was a postdoctoral fellow at Beijing Computational Science Research Center (CSRC) from 2016 to 2019. Since 2020, he has worked as an associate professor at TIPC, CAS. His current research focuses on first-principles calculations for nonlinear optical materials.

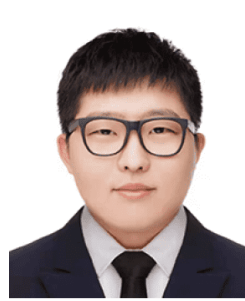

Fei Liang received his BSc in physics from Nanjing University, and PhD in materials science from TIPC, CAS in 2019. Then, he joined the Institute of Crystal Materials, Shandong University. His current interest focuses on the structural design and property characterization of laser crystals and nonlinear optical crystals.

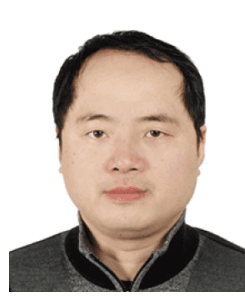

Zheshuai Lin received his BSc in physics from Tianjin University, and $\mathrm{PhD}$ from Fujian Institute of Research on the Structure of Matter, CAS in 2002. From 2004 to 2008, he was a research associate at Cambridge University, UK. Since 2008, he has worked as a research professor in TIPC, CAS. His research into nonlinear optical crystals employs a variety of modeling techniques spanning analytical and quantum mechanics, as well as experimental exploration.

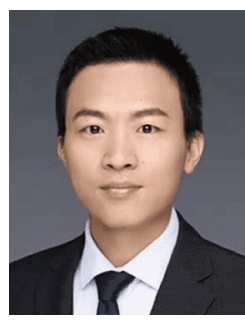

Bing Huang received his BS in physics from Jilin University, and $\mathrm{PhD}$ in condensed matter physics from Tsinghua University in 2010. Between 2010 and 2015 , he was a post-doctoral or research fellow at National Renewable Energy Lab, Oak Ridge National Lab and University of Utah. Since 2016, he has worked as a tenure-track professor at CSRC and an adjoint professor at Beijing Normal University. His current research focuses on computational semiconductor physics.

\section{深紫外非线性光学晶体设计：基于第一性原理的} 计算机辅助设计蓝图

康雷 $^{1,2}$, 梁飞 ${ }^{1}$, 林哲帅 ${ }^{1 *}$, 黄兵 ${ }^{2 *}$

摘要 深紫外非线性光学晶体在当前深紫外全固态激光技术中是 不可或缺的, 它是通过频率转换产生深紫外相干光的核心元件. 由 于严苛的性能标准, 深紫外非线性光学晶体非常稀少, 其新型材料 的发现面临巨大挑战. 尽管 $\mathrm{KBe}_{2} \mathrm{BO}_{3} \mathrm{~F}_{2}(\mathrm{KBBF})$ 晶体对于许多当前的 用途已经非常出色，但是深紫外科学的发展需要深紫外非线性光 学晶体具有更短的输出波长和更大的倍频效应, 以满足更高精度 和更高功率的深紫外激光需求. 因此, 如何有效地设计深紫外非线 性光学晶体一直是非线性材料科学领域的一个核心问题. 回顾非 线性材料的发展, 理论设计和模拟对非线性光学机理的理解和非 线性材料的探索都是一种有效且高效的方案. 为了进一步加速深 紫外非线性材料的探索进程, 在本文中, 我们总结并提出了一套功 能全面的计算机辅助建模系统和设计蓝图, 该系统可用于高效且 严格地评估候选材料的深紫外非线性光学性能. 依靠此建模系统, 我们不仅可以丰富对非线性光学结构与性能关系的理解, 而且能 够从许多已知的和设计的结构出发，根据不同的结构类型和化学 组分, 系统研究其深紫外非线性光学的具体特性. 基于此设计蓝图, 我们总结了二十多种可能的深紫外非线性光学晶体结构, 并首次 预言了能够呈现出潜在的深紫外非线性光学性能的七种新颖结构. 我们相信, 这套计算机辅助建模系统和设计蓝图将会在探索新型 深紫外非线性光学材料的进程中发挥重要作用. 